\title{
Le Parlement italien : l'exemple d'un bicamérisme plus que parfait sans équivalent
}

\author{
Adriano EVANGELISTI \\ Doctorant en droit public \\ Université d'Aix en Provence \\ ILF/GERCJ UMR 7318, ED 67
}

La solution retenue par les constituants italiens d'adopter un bicamérisme égalitaire dans la constitution de 1948 se justifiait car ce dernier était majoritaire dans les pays européens de l'époque. En effet, seulement 4 pays avaient mis en place un bicamérisme inégalitaire : Autriche, Angleterre ${ }^{1}$, Irlande, Grèce.

Si le bicamérisme mis en place s'apparentait à celui de la Constitution Belge de 1827 qui avait énormément influencé la rédaction du Statut Albertin, les constitutions des autres pays européens et les évolutions constitutionnelles successives s'affranchirent du bicamérisme égalitaire en mettant en place différentes formes de rationalisation.

La raréfaction progressive du bicamérisme égalitaire nous permet de dire que le modèle italien est aujourd'hui somme rare.

C'est alors que le droit comparé nous permet de saisir la singularité du bicamérisme italien qui réside dans le fait que ce dernier est le plus égalitaire parmi les bicamérismes égalitaires contemporains (II) et passés, notamment celui

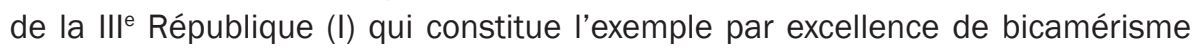
parfait.

1 En se référant à l'Angleterre, ce pays a codifié la primauté de la chambre basse de son Parlement à travers les Parliament Acts de 1911 et 1949. Il est intéressant d'observer comment le Parlement pré-républicain italien a mis en place une règle du même ordre sans que celle-ci ne soit codifiée, un genre de Informal Parliament Act. En effet, sans altérer les prérogatives de l'ancien Sénat, le mécanismes des infournades de sénateurs avait pour finalité d'annuler le pouvoir de blocage d'un Sénat à nomination royale. 


\section{Le bicamérisme italien et français de la III ${ }^{\mathrm{e}}$ République}

La nature égalitaire de ce bicamérisme constitue un point commun avec le système mis en place par la Constitution de 1947. Si l'exercice conjoint de la fonction législative est l'aspect principal qui caractérise l'égalitarisme des deux chambres, il n'en est pas de même pour d'autres prérogatives. Ainsi, le bicamérisme de l'époque s'apparente-t-il à un bicamérisme asymétrique, contrairement à son homologue italien qui se caractérise par une symétrie presque parfaite (A).

Si les deux bicamérismes ont une deuxième chambre qui, de iure, est sur le même pied d'égalité concernant la fonction législative, le Sénat français de la III a exercé sa fonction de chambre de réflexion, souvent comme Chambre de blocage, contrairement au Sénat italien (B).

En effet, la mise en place du Sénat italien s'affranchit de la mise en place évoquée en France où le Sénat, objet d'une loi constitutionnelle ad hoc, constitue le gage ou la concession des Républicains aux monarchistes ${ }^{2}$. L'ambiguïté des positions des constituants ne permet pas de saisir immédiatement le rôle effectif de la deuxième chambre qui aurait dû avoir une fonction différente de la première (Chambre des Régions ou des catégories professionnelles) et qui, à la fin, en raison de l'attribution de compétences identiques et de son élection au suffrage universel, s'est réduite à une copie de la Chambre des députés. Le seul élément de distinction avec la première chambre résidait dans l'amélioration de la législation, qui figure dans le rapport qui accompagne le premier texte de Constitution à l'Assemblée Constituante.

\section{A. Une différente symétrie bicamérale}

Les régimes des asymétries/symétries du bicamérisme présentes sous la III République permettent une répartition des compétences entre les deux chambres du Parlement qui demeure absente s'agissant de la fonction législative en raison de la participation concurrente du Sénat "à l'initiative et à la confection des lois ", (art. 8, al. 1 loi constitutionnelle du 24 février 1875).

Si l'exercice de la fonction législative demeure partagé, l'initiative législative, qui est aussi exercée de manière concurrente par les deux chambres, ne demeure pas pleinement égalitaire pour les lois des finances en raison de la priorité accordée à la Chambre des députés pour l'initiative législative et pour le vote en première lecture (alinéa 2 du même article). Si cette priorité d'examen et de vote s'apparente au principe libéral " no taxation without representation ", cette similitude ne va pas plus loin en raison des mêmes compétences législatives attribuées au Sénat lors de l'examen parlementaire, réduisant le principe à un simple détail de forme : la priorité dans le dépôt et le vote.

2 Cf. R. DEBBASCH, Droit Constitutionnel, 11e édition, Lexis Nexis, Novembre 2018, op. cit., p. 109. 
L'asymétrie entre les deux chambres vise le deux compétences octroyées au Sénat : la possibilité de se réunir en Cour de Justice pour juger le président de la République et les Ministres, ainsi que tout citoyen portant atteinte à la sécurité de l'État ; l'avis conforme pour dissoudre la Chambre des députés.

Le premier de ces pouvoirs, qui vise la responsabilité politico-criminelle du président de la République et des Ministres, s'apparente à l'impeachment américain : fonction accusatrice par la Chambre des députés, fonction de jugement exercée par le Sénat. De manière similaire à l'exemple américain, la notion " d'attentats contre la sûreté de l'État " est suffisamment large, permettant au Sénat de s'affranchir des normes contenues dans le code pénal et d'englober dans la notion d'attentats contre la sûreté étatique de simples complots.

Le pouvoir accordé au Sénat en matière de dissolution parlementaire, sur proposition du président de la République, est une des prérogatives les plus frappantes constituant la divergence majeure du bicamérisme égalitaire prévue par l'article 8 de la $2^{\mathrm{e}}$ loi constitutionnelle de 1875 relative à l'organisation du Sénat. La singularité de la norme contenue dans l'article 8 place sur un pied inégalitaire les deux chambres, en raison de la dissolution qui n'est applicable qu'à l'endroit de la Chambre des députés, et de surcroît avec le consentement du Sénat ${ }^{3}$. Ce qui permet de constater le pouvoir accordé à une chambre non élue au suffrage universel direct, qui de son côté est indissoluble, de dissoudre la chambre élective. Le Sénat, qui devait être le compromis pour l'institution de la III République, constituait l'institution parlementaire conservatrice nécessaire à contrecarrer une chambre des députés élue au suffrage universel. La mise en place d'une dissolution se justifiait en présence des majorités parlementaires opposées, notamment celle de la Chambre des députés.

La procédure de dissolution activable sur proposition du président de la République et avec l'aval du Sénat, mettait en crise la conception égalitaire de ce bicamérisme : en effet, il était difficilement concevable que l'exercice collectif de la fonction législative puisse avoir lieu si une des deux chambres pouvait être dissoute sur l'avis de l'autre. En outre, sa mise en place constitue une prérogative discrétionnaire du président de la République vu l'absence de motifs pouvant la justifier et son recours illimité. L'article 5 contenait donc les prémices pour un bicamérisme et un rapport des pouvoirs conflictuels, c'est ainsi que la pratique (crise du 16 mai 1877) a fait tomber en désuétude le pouvoir de dissolution.

Concernant le pouvoir de révision constitutionnelle (article 8 de la $1^{\text {re }}$ loi constitutionnelle de 1875), sa procédure hybride ne prévoit pas de procédure égalitaire. En effet, si initialement les deux chambres exerçaient concurremment

3 En reprenant la terminologie utilisée par Julien Boudon dans Bicamérisme ou bicamérismes ? Essai de Typologie des Parlements Bicaméraux, Historia lus, rivista di storia giuridica dell'età medioevale e moderna, le bicamérisme existant sous la Illème s'apparentait à un bicamérisme presque parfaitement égalitaire, op. cit., p. 6, avec une prédominance de la deuxième chambre, avant que la disposition contenue dans l'article 5 de la $1^{\text {re }}$ loi constitutionnelle tombe en désuétude. 
le pouvoir de révision au moyen de délibérations prises séparément à la majorité absolue des voix, après cette première délibération la procédure de révision devenait effective par une réunion conjointe des deux Assemblées (Assemblée Nationale) statuant à la majorité absolue des membres. Ainsi, l'égalité des deux chambres qui s'exerçait initialement était-elle réduite par la séance conjointe des deux Assemblées qui plaçait sur un pied d'inégalité les sénateurs et les députés, en raison des effectifs inférieurs.

La symétrie presque parfaite du bicamérisme italien se traduit par une évolution du bicamérisme existant sous la III e en attribuant aux deux chambres l'exercice des mêmes compétences ; pour cette raison, le bicamérisme est parfaitement égalitaire et constitue l'exemple le plus pur de bicamérisme égalitaire ${ }^{4}$.

Le fondement majeur de cet égalitarisme réside dans l'article 70 de la Constitution "la fonction législative est exercée collectivement par les deux chambres ". Le mot collectivement s'est traduit par un exercice conjoint mais séparé de la fonction législative. Au premier abord, on pourrait se questionner sur la portée de l'article 70, notamment sur sa mise en place : de quelle manière se met en place l'exercice collectif de la fonction législative par les deux chambres? En effet, l'adverbe collectivement renvoie à un exercice conjoint. Aussi, il serait possible d'envisager la possibilité que l'exercice collectif de la fonction législative exercée par les deux chambres se traduise par une séance conjointe, attribuant ainsi à l'adverbe collectivement la portée d'exercice conjoint. La portée de l'article 70 de la Constitution est éclaircie par le deuxième alinéa de l'article 55 de la Constitution qui prévoit que " Le Parlement siège en chambres réunies dans les seuls cas établis par la Constitution " et l'article 72.

La Constitution prévoit ainsi la séance conjointe des deux assemblées dans 5 circonstances distinctes : l'élection du président de la République (articles 83 et 85), la mise en accusation du président de la République (art. 90), le serment du président de la République (art. 91), l'élection d'1/3 des membres du Conseil Supérieur de la Magistrature (art. 104), l'élection d'1/3 des membres de la Cour Constitutionnelle (art. 135). La séance conjointe des deux Assemblées pour exercer collectivement la fonction législative ne rentre pas dans les cas prévus par l'alinéa 2 de l'article 55. Une telle séance conjointe contredirait la Constitution et entraînerait une déclaration d'inconstitutionnalité par la juridiction constitutionnelle italienne, puisque l'article 70 ne prévoit pas que " la fonction législative est exercée collectivement par les deux chambres réunies à cet effet en séance conjointe ".

Enfin, la portée de l'exercice collectif réside dans l'alinéa 2 de l'article 72, puisqu'il est prévu que " tout projet de loi présenté à l'une des deux chambres est aux termes de son règlement, examiné par une commission puis par la chambre elle-même ". Les termes utilisés " une des deux chambres ", " chambre elle-même "

4 Cf. J. BOUDON, Idem. 
ne laissent aucun doute sur l'exercice séparé de la fonction législative, à mettre en place selon les normes prévues dans le règlement de chaque chambre.

L'égalité des deux chambres italiennes englobe aussi la fonction de contrôle de l'exécutif (art. 94 de la Constitution).

Contrairement à l'article 70 qui prévoit littéralement un exercice collectif de la fonction législative, le pouvoir de contrôle est exercé séparément, comme en témoigne l'alinéa 2 de l'article 94 : "Chaque chambre accorde ou refuse la confiance au moyen d'une motion motivée et votée par appel nominal ". La confiance parlementaire, obligation pour que l'exécutif entre dans la plénitude de ses fonctions, n'est jamais présumée comme le prévoit l'alinéa 1 de l'article 94 puisque "Le Gouvernement doit avoir la confiance des deux chambres ".

Ainsi, les deux chambres accordent la confiance initiale à l'exécutif dans un délai constitutionnellement contraignant, " dans les 10 jours qui suivent la formation du gouvernement ", et peuvent la lui révoquer ou la lui renouveler à tout moment.

L'attribution de compétences identiques dans l'octroi ou la révocation de la confiance à l'exécutif met en place un régime de confiance parfaite entre les deux chambres ; la confiance est indispensable pour que le gouvernement reste en fonction.

Si un tel régime est prévu par la Constitution, la majorité des crises de gouvernement reste de nature extra parlementaire, ${ }^{5}$ alors qu'une crise de gouvernement implique qu'elle soit reconduite dans les institutions parlementaires $^{6}$ (parlamentarizzazione della crisi). En effet, les crises extra parlementaires nécessitent une désapplication de l'article 94 de la Constitution qui se traduit dans les faits par une marginalisation du Parlement en faveur d'un régime partitocratique. Deux cas dans I'histoire constitutionnelle italienne ont constitué une exception à la pratique dérogratrice de l'article 94 : les deux crises du gouvernement Prodi I (1996-1998) et II (2006-2008).

La crise du Gouvernement Prodi II a constitué un cas de crise parlementaire parfaite $^{7}$, en raison de l'application constitutionnellement irréprochable de l'article 94 : le président du Conseil suite à le démission de son ministre de la Justice se présenta devant le Parlement en vue d'un vote de confiance qui lui fut accordé par la Chambre des députés (326 voix pour, 275 voix contre) mais rejeté par le Sénat (161 voix pour, 156 voix contre). Néanmoins, ces deux cas ont constitué des exceptions, les gouvernements successifs n'ont pas suivi l'exemple

5 Dans ces circonstances les crises politiques se réduisaient à une crise au sein de la majorité gouvernementale qui n'investissait pas le Parlement : le premier Ministre ne demandait pas un vote au Parlement et présentait directement sa démission au président de la République.

6 Une telle préoccupation faisait l'objet en 1990 d'une proposition de loi constitutionnelle présentée par le président de la Chambre des députés de l'époque, Oscar Luigi Scalfaro. Cette proposition prévoyait l'obligation pour le gouvernement qui avait l'intention de démissionner d'informer les chambres au moyen de déclarations dûment motivées, devant faire l'objet d'un vote parlementaire. Cf. A.C N.5231, Xe lègislature, Actes parlementaires.

7 Cf. V. LIPPOLIS, "La crisi del Secondo Governo Prodi II ", Federalismi n.3/2008, art. cit., p. 1. 
des deux gouvernements Prodi ${ }^{8}$.

Si on considère la place importante que les constituants ont voulu réserver au Parlement, les crises extra parlementaires sont un vulnus pour le rôle qu'exerce constitutionnellement le Parlement envers les exécutifs, et constituent un cas de désuétude constitutionnelle de l'alinéa 2 de l'article 94 qui se traduit par la mise en place d'une convention contra constitutionem. Celle-ci met en crise l'exercice paritaire des deux chambres de révoquer la confiance à l'exécutif et place le Parlement dans une zone d'ombre au profit des organes des partis où se décident les sorts des gouvernements. La reconduite d'une crise dans les enceintes parlementaires doit répondre à des exigences de clarté et de transparence face à l'opinion publique. Ces dernières se mettent en place par l'alinéa 2 de l'article 94 prévoyant que l'octroi ou l'accord de la confiance parlementaire au gouvernement ait lieu par appel nominal. La solution des crises en dehors de l'institution parlementaire prive cette dernière de l'une de ses fonctions primaires et porte atteinte aux exigences de clarté et de transparence envers les électeurs , qui doivent être mis en condition de connaître les raisons pour lesquelles est révoquée la confiance au gouvernement. Seul le Parlement permet de garantir ces deux exigences.

L'attribution des mêmes compétences en matière de contrôle à l'exécutif fut à la fin retenue par les constituants pour préserver la nature égalitaire du bicamérisme.

À l'origine, le premier projet de constitution italienne (31 janvier 1947) prévoyait une rationalisation du rapport de confiance en vertu de l'o.d.j Perassi'. Concernant la confiance initiale à l'exécutif (alinéa 3 article 94 de la constitution), cette dernière était accordée conjointement par les deux Assemblées réunies en Assemblée Nationale. La confiance était accordée au moyen d'une motion à la majorité absolue des membres ( $2^{\mathrm{e}}$ et $3^{\mathrm{e}}$ alinéa de l'art. 87).

Les chambres avaient la faculté de révoquer la confiance par une motion de défiance (art. 88 alinéa 2 du projet de Constitution) qui pouvaient être présentée

8 Le Gouvernement Berlusconi IV va présenter sa démission suite aux tensions internes à la majorité. Le Gouvernement Monti I démissionna le 12 décembre 2012 suite à un vote d'abstention d'un parti soutenant l'exécutif. Le Gouvernement Letta I (2013-2014) démissionna suite à un vote de la direction du Parti démocrate (organe interne du Parti, constituant une sorte de parlement interne du groupe politique) qui demandait un changement à la tête de l'exécutif. Le Gouvernement Renzi I (2014-2016) constitue un cas emblématique car le Premier Ministre démissionna suite au rejet de son projet de révision constitutionnelle (4 décembre 2016) alors même que la majorité parlementaire soutenant l'exécutif était toujours présente. Le Gouvernement Gentiloni I (2016-2018) se place dans un cas de démission obligée en raison de la fin de la législature. Le Gouvernement Conte I (2018-2019) se situe dans une circonstance particulière car l'exécutif le Premier Ministre se présenta au Sénat pour tenir un discours dans lequel il expliquait les raisons de la fin de son gouvernement. Pour plus de détails, A. EVANGELISTI, "Le droit parlementaire: clé de la crise du gouvernement ", Civitas Europa, 2019/2 ( $\left.{ }^{\circ} 43\right)$.

Voir supra, p. 4, note 1. 
selon les mêmes critères prévus par l'actuel dernier alinéa de l'article 94. Jusqu'ici le mécanisme se rapprochait du régime prévu par l'art. 94. Toutefois l'art. 88 prévoyait un mécanisme de rationalisation singulier permettant au gouvernement, après le vote de défiance par une chambre, de ne pas démissionner et de demander un nouveau vote par le Parlement réuni en Assemblée Nationale. La rationalisation se mettait en place par trois critères : la confiance initiale qui était accordée par le Parlement en séance commune ; la possibilité de réunir le Parlement en séance commune pour faire appel d'une décision prise séparément par une des deux chambres ; l'absence de quorum pour le vote de l'Assemblée Nationale.

Un tel mécanisme était conçu pour réduire le poids des chambres grâce au vote initial de confiance et au vote d'appe $/^{10}$ en Assemblée Nationale.

La logique de ce mécanisme de confiance reposait sur la centralité de l'Assemblée Nationale. Considérant que cette dernière investissait l'exécutif par un vote initial, seule celle-ci était en mesure de le perverser. Toutefois il était difficile de concevoir un vote d'appel après que l'une des deux chambres ait votée une motion de défiance. En outre, quelle était la portée d'une telle motion si le Gouvernement pouvait demander un vote d'appel par l'Assemblée Nationale?

Enfin, il est important d'observer que tel mécanisme aurait impliqué une paralysie législative. En effet, par le vote d'appel de l'Assemblée Nationale, l'exécutif aurait pu obtenir la majorité en raison des différents effectifs numériques des deux Assemblées qui privilégiaient la chambre des députés. Toutefois, en raison du bicamérisme égalitaire, le concours de la chambre qui il ne disposait plus de la majorité, lui, était indispensable afin de mener sa politique législative et son obstruction pouvait alors entraîner une paralysie de la procédure législative.

Le mécanisme du vote appel fut donc éliminé de même que le vote de confiance accordé en Assemblée Nationale en raison des effectifs numériques différents qui plaçaient dans une position inégalitaire les sénateurs par rapport aux députés.

C'est ainsi que l'égalité des fonctions en matière de contrôle de l'action du gouvernement (art. 94) entraîna une méconnaissance des propos contenus dans l'o.d.j Perassi, mise en place par une élimination de mécanismes constitutionnels conçus pour établir une rationalisation de la forme de gouvernement.

Les deux chambres exercent aussi des fonctions de manière conjointe lorsqu'elles se réunissent en séance commune du Parlement pour élire le président de la République (art. 83) et le mettre en état d'accusation (art. 90), et lors de l'élection d'1/3 des membres de la Cour Constitutionnelle (art. 135) et du CSM (art. 104), la rédaction tous les 9 ans de la liste des 45 citoyens pouvant

10 On utilise cette définition car l'article 88 dernier alinéa du projet prévoyait : " Après qu'une motion de défiance est votée par une des deux chambres le Gouvernement, s'il n'a pas l'intention de démissionner, doit convoquer l'Assemblée Nationale qui se prononce au moyen d'une motion ". Par la formule employée " s'il n'a pas l'intention de démissionner ", on observe bien un appel activable à discrétion de l'exécutif. 
être élus sénateurs qui vont intégrer la Cour Constitutionnelle (membri aggregati), dans le jugement du président de la République (art. 135 dernier alinéa) et la participation au serment de fidélité du président de la République récemment élu (art. 91).

Concernant ses fonctions, la tendance serait celle d'imaginer qu'il y a une égalité aussi patente ${ }^{11}$. Néanmoins, il faut préciser que le concept d'égalité patente renvoie à l'exercice de la fonction législative qui est faite de manière paritaire entre les deux chambres, conformément à l'article 70, et à l'octroi ou révocation de la confiance au Gouvernement (article 94 alinéa 2). Concernant le Parlement en séance commune, ce dernier n'exerce pas de fonctions législatives mais des fonctions encadrées par la Constitution. Pour cette raison, il s'agit d'un organe constitutionnellement différent, en vertu de ses fonctions et de sa composition, par rapport à celui qui agit en vertu de l'article 70 .

Le Parlement en séance commune est un cas sui generis qui exerce des fonctions électorales (articles 83, 104, 135 alinéa 1 et 135 dernier alinéa), des fonctions d'accusation (article 90) et des fonctions d'admission ou de prise d'acte (funzione ricettizia) s'agissant du Parlement réuni en séance commune pour le serment de fidélité du président de la République (art. 91) ${ }^{12}$.

Le Parlement en séance commune constituerait un cas de collège électoral parfait et imparfait en fonction des différences qui lui sont attribuées par la Constitution.

S'agissant des fonctions électorales, on serait face à un collège électoral imparfait en raison de l'impossibilité pour ce dernier de pouvoir discuter, mais d'accomplir des fonctions électives (élection des juges constitutionnels, juges du CSM, du président de la République).

En revanche, s'agissant de la mise en accusation du président de la République, on serait face à un collège électoral parfait, puisque la délibération sur la mise en accusation serait précédée d'une discussion préliminaire finalisée afin de savoir si les conditions sont retenues pour pouvoir délibérer.

Pour le dernier cas (art. 91), le Parlement en séance commune n'exercerait pas de fonction comparable à un collège électoral, il n'aurait aucune fonction active mais vérifierait qu'a lieu le serment de fidélité du Président élu selon les conditions prévues par la Constitution.

Le concept d'égalité patente est lié à un exercice paritaire des compétences par les deux chambres, lequel peut avoir lieu en raison du fait que celles-ci sont sur le même plan d'égalité. La séance commune du Parlement ne satisfait pas ces exigences paritaires en raison des effectifs numériques qui placeraient une assemblée (le Sénat) en position inégalitaire par rapport à l'autre.

C'est ainsi que le Parlement en séance commune constitue un cas à part, en dehors du régime bicaméral italien.

11 Cf., J. BOUDON, op. cit.

12 Cf. S. CICCONNETTI, Diritto Parlamentare, Giappichelli, Torino, op. cit., p. 261. 
La nature égalitaire du bicamérisme, qui se traduit aussi par l'élection des deux chambres au suffrage universel, est présente également au niveau du pouvoir de dissolution qui peut frapper les deux chambres. Ainsi, une des conséquences du bicamérisme égalitaire est la dissolution (de facto) égalitaire. Comme l'a souligné une part de la doctrine ${ }^{13}$, il s'agit d'un des aspects les plus originaux du bicamérisme italien car l'Italie est le seul pays d'Europe où le Sénat peut faire l'objet d'une dissolution ${ }^{14}$.

Une autre fonction exercée de manière paritaire concerne la procédure de révision constitutionnelle qui toutefois voit toujours le concours symétrique des deux chambres car la procédure de révision des constitutions modernes est toujours aggravée, faute de quoi il s'agirait de constitutions flexibles. La procédure de révision prévue par l'article 138 est une des procédures les plus faciles par rapport à d'autres existantes dans d'autres pays.

Sur ce point seulement, la France a une procédure constitutionnelle plus souple en raison des quorum requis (majorité simple) et du pouvoir discrétionnaire attribué au président de la République de convoquer le Parlement en Congrès qui, en cas d'adoption du texte à la majorité des $3 / 5$ des suffrages exprimés, permet d'éviter le référendum.

La symétrie presque parfaite du bicamérisme italien précédemment évoquée,

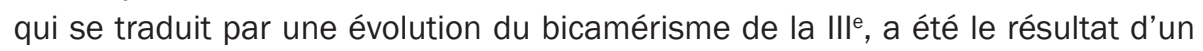
choix non prémédité en raison du voile d'ignorance présent au moment de la décision définitive. Ainsi, une évolution du système parlementaire présent en France au moment de la $\mathrm{II}^{\mathrm{e}}$ s'apparente au bicamérisme suédois en vigueur jusqu'à 1969.

Les éléments existants entre les deux chambres, qui ne permettent pas de parler de symétrie parfaite, résident essentiellement dans les effectifs numériques et dans l'électorat distinct pour élire les députés et sénateurs (18 ans pour les députés, 25 ans pour les sénateurs). C'est surtout ce dernier aspect qui permet de cerner la différence majeure entre les deux chambres.

$\mathrm{Si}$, initialement, la différence pour élire les deux chambres se réduisait à 4 ans en raison de la majorité fixée à 21 ans, l'abaissement en 1975 de la majorité à 18 ans a entraîné une différence de 7 ans entre les deux électorats. Cette différence en terme d'électorat se traduit en environ 4 millions d'électeurs ${ }^{15}$ qui sont exclus du vote pour le Sénat.

On observe ainsi que si la majorité est fixée à 18 ans, pour l'élection des deux chambres il y a deux majorités électorales pour élire les deux chambres ${ }^{16}$ : une première majorité fixée à 18 ans, une deuxième fixée à 25 ans.

La différence des électorats pour les deux chambres est évoquée souvent

13 Cf., A. GIACONE, "Le Sénat italien ou le bicamérisme égalitaire dans La Chambre Haute Hier en France ", Aujourd'hui en Europe, Jean-Marc Guislin éditeur, IRHS, cit., p. 158.

14 Cf., A. GIACONE, Idem.

15 Chiffres extraits par le site du Ministère Intérieur Italien.

16 Cf., A. GIACONE, art. cit., p. 159. 
par la doctrine comme un aspect anachronique qui n'a plus lieu d'être ${ }^{17}$ et nécessitant un alignement, 18 ans pour élire les membres des deux chambres, et pouvant conduire à la formation de majorités opposées entre les deux chambres, empêchant ainsi le fonctionnement du bicamérisme.

Un tel phénomène se produisit lors des élections de 2006 où, au Sénat le centre gauche avait une majorité quasi inexistante (157 voix contre 156). Néanmoins, il faut constater qu'une telle circonstance n'était pas due aux électorats hétérogènes des deux chambres mais à la législation électorale de l'époque (loi 270/2005), déclarée ensuite inconstitutionnelle (jugement n. 1/2014), qui avait rompu l'homogénéité existante entre les deux chambres en prévoyant un scrutin asymétrique pour élire les deux chambres : un bonus majoritaire à l'échelle nationale pour la Chambre des députés, des bonus majoritaires à l'échelle de chaque région. Avant l'approbation de la loi 270/2005, les électorats distincts entre les deux chambres ne furent pas à l'origine d'hétérogénéités significatives concernant la composition des deux chambres. C'est pour cette raison que si, de iure, la question put paraître constitutionnellement bien fondée, de facto cette différence n'a jamais produit de majorités opposées en raison de la structure nationale des partis politiques italiens ${ }^{18}$.

La réforme des électorats fit l'objet d'un examen du Parlement sous la XVIII législature, notamment du Sénat. Le texte approuvé par ce dernier prévoit un alignement total entre le critère d'âge (elettorato attivo) et le critère d'éligibilité (elettorato passivo) pour élire les députés et sénateurs : 18 ans pour élire les parlementaires, 25 ans pour être élus ${ }^{19}$. En raison de l'exercice des mêmes compétences, il est opportun que les électorats qui élisent cette chambre soit identiques à ceux qui élisent la chambre des députés. Pour cette raison, une symétrie sur les critères d'âge paraît suffisante. En effet, une asymétrie de l'elettorato attivo pourrait fausser l'élection du Sénat en raison de l'exclusion de 4 millions d'électeurs, notamment en présence d'une coalition obtenant la majorité avec une différence en terme de voix risible.

Toutefois, l'alignement de l'elettorato passivo pour les sénateurs à celui des députés est peu opportun et même problématique. En effet, une symétrie parfaite entre l'elettorato attivo et passivo mettrait en place un bicamérisme hyperégalitaire (bicamerismo perfettissimo) où la seule différence entre les deux chambres viserait les effectifs numériques. L'alignement de l'elettorato passivo pour les sénateurs éliminerait la seule différence possible en terme de composition pour le Sénat : celui de représenter une assemblée dont les membres sont plus âgés. En outre, l'alignement du critère d'éligibilité pour les sénateurs à celui des députés aurait l'effet de contredire l'essence du mot sénateur (senex, plus âgés), mettant en place un cas sui generis de Sénat car, dans tous les pays

17 Voir N. LUPO, S. CECCANTI, A. MANZELLA.

18 Cf., N. LUPO, Corso di diritto Parlamentare, IL Mulino, Bologna, op. cit., p. 118.

19 A.C. $224 \mathrm{XVIII}$ legislature, site chambre des députés. 
où les sénateurs sont élus au suffrage universel direct ${ }^{20}$ ou indirect, ces derniers ont toujours un âge plus avancé que leurs collègues députés. Ce projet mettrait en place une réelle chambre doublon avec fonctions et composition parfaitement identiques qui constituerait véritablement un cas de monocamérisme caché sous l'apparence constitutionnelle d'un bicamérisme parfait. Une réforme de ce genre serait peu opportune et rendrait encore plus encombrant et inutile le bicamérisme égalitaire.

C'est pour ces raisons, à moins qu'il soit envisagé de modifier la nature du bicamérisme, qu'il paraît judicieux de préserver les petites asymétries encore existantes dans le bicamérisme égalitaire italien.

\section{B. Deux types de bicamérismes différemment égalitaires}

Si de iure le bicamérisme de la III $^{\mathrm{e}}$ République et celui italien impliquent un concours paritaire du Sénat à la fonction législative, de facto l'ancien Sénat français utilisa les mêmes prérogatives en matière législative à des fins d'enlisement et retardement de l'approbation des textes. Ainsi il agissait comme chambre de blocage plutôt que chambre de réflexion. Une conduite qui s'explique par sa composition conservatrice contrairement au Sénat italien qui, même en ayant des attributions identiques à la Chambre des députés, est issue du suffrage universel direct. Une telle nature élective identique empêche cette chambre de se positionner comme chambre conservatrice puisqu'elle est élue quasiment par les mêmes électeurs. De iure, on pourrait objecter aujourd'hui sa nature conservatrice en raison de l'exclusion des électeurs entre 18 et 25 ans.

Les navettes parlementaires permettent de saisir la fonction exercée par le Sénat italien (1) et celle du Sénat de la III République (2).

\section{Le Sénat italien dans la procédure législative}

Dans la doctrine italienne s'est répandue depuis plusieurs années l'idée que le bicamérisme égalitaire, souvent qualifié de bicamérisme répétitif, est une perte de temps considérable car, entre le dépôt initial d'un texte et son approbation définitive par les deux chambres, s'écoulent de nombreuses années ${ }^{21}$. Une

20 En Europe les sénateurs sont élus au suffrage universel direct encore en Pologne et en République Tchèque. Dans le premier pays les sénateurs sont élus par les électeurs ayant 18 ans (elettorato attivo), l'âge d'eligibilité est fixé à 30 ans. Dans le deuxième, l'elettorato attivo est le même alors que l'âge d'éligibilité est fixé à 40 ans. On constate ainsi comme il y a une asymétrie entre l'elettorato attivo e passivo.

21 La constitution italienne ne donne aucune indication temporaire concernant les lectures parlementaires, contrairement à la constitution française qui, depuis la révision de 2008, prévoit à son article 42 alinéa 3, délais a minima pour l'examen du texte à compter de son dépôt : 6 semaines en $1^{\text {re }}$ lecture, 4 semainesà compter de la $2^{\mathrm{e}}$ lecture. La constitution se limite à esquisser des modalités d'examen pour chaque lecture (article 72 alinéa 1). C'est ainsi que, de iure, la procédure législative à une durée indéterminée dans son ensemble et dans chaque lecture parlementaire. Pour ces raisons on peut affirmer que certes la procédure peut demeurer longue 
telle affirmation, qui juridiquement est fondée, doit être analysée avec les lois approuvées.

En faisant une analyse des lois approuvées depuis 1948 (graphique $1^{22}$ ), on constate que la majorité de celles-ci ont été approuvées après seulement deux lectures parlementaires.

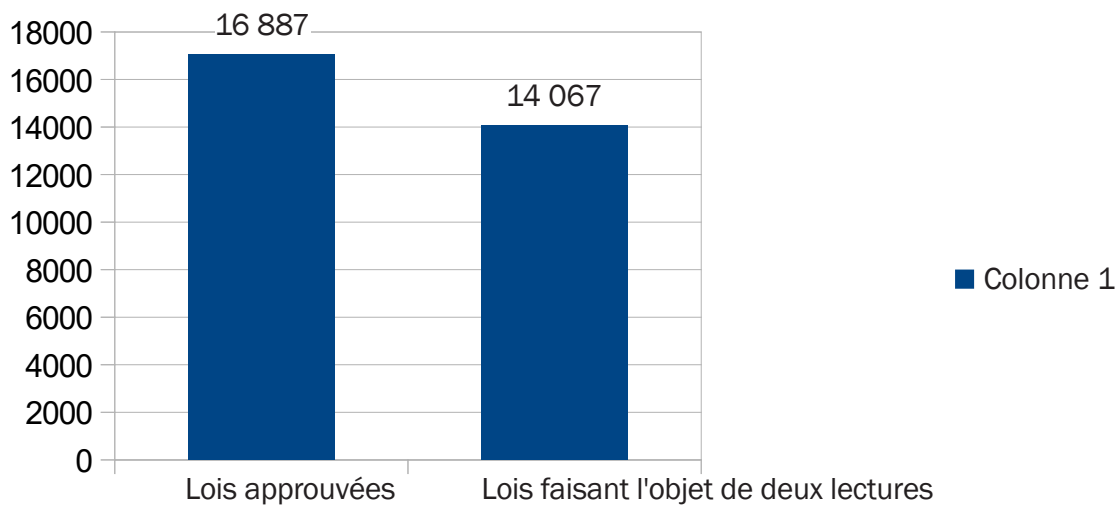

Plus précisément, de la Ire législature (1948-1953) jusqu'à celle en cours $(\mathrm{XVIII})$ ), le Parlement italien a voté 16887 lois (dont 14067 qui ont été approuvées après seulement 2 lectures, soit 1 seule navette). Ainsi, $83 \%$ des lois votées n'ont fait l'objet d'aucune navette parlementaire, ce qui constitue un des atouts du bicamérisme. Concernant les autres lois, elles se répartissent de la manière suivante : 2375 lois ont été approuvées après 3 lectures, $13.9 \%$ des lois approuvées ; 366 lois ont fait l'objet de 4 lectures (2.1\%) et 14 de 6 lectures.

Les deux concepts, navette parlementaire et lectures parlementaires, ne doivent pas être confondus. En raison du bicamérisme, la procédure normale prévoit une double approbation nécessaire par les deux chambres sur le même texte qui se met en place par les lectures parlementaires. Toutefois, après la première lecture, si la deuxième chambre introduit des modifications, le texte doit revenir à la chambre initiale (navette) qui pourra confirmer le texte voté par la deuxième assemblée ou apporter des amendements qui devront faire l'objet d'un autre passage parlementaire, jusqu'à ce que l'on parvienne à une approbation en termes identiques par les deux chambres. Lorsqu'une loi fait l'objet de deux

mais peut, par ailleurs, être plus courte en raison de l'absence de délais temporaires lors des différentes lectures. II faut toutefois relativiser la présence de ces délais en raison de la normalisation de la procédure accélérée prévue à l'article 45 et de ses effets (dernier alinéa de l'article 43) qui annulent les délais précédemment mentionnés.

22 Les données ont été extraites par le site de legislature precedenti de la Chambre des députés italienne, (https://legislatureprecedenti.camera.it/), qui contient l'ensemble de la législation adopté dans chaque législature républicaine. Dans ces statistiques ont été prises en compte uniquement les lois approuvées selon la procédure législative prévue aux articles $70,71,72$ de la Constitution. Les lois régionales n'ont pas été prises en compte en raison du régime monocaméral présent dans les 20 Régions italiennes. 
lectures parlementaires, il n'y a pas de navette. En approuvant le texte de manière identique, la deuxième chambre met ainsi fin à la procédure législative.

Hormis le cas des lois faisant l'objet d'une seule navette, les autres lois qui ont fait l'objet de plusieurs navettes restent assez rares. Le cas le plus emblématique est constitué par la loi 338 du 9 avril 1953 dispositions pour la gestion financière des services des préventions des incendies qui reste la seule loi qui a été approuvée le 15 mars 1953, après 8 lectures parlementaires, soit 3 navettes en totalité et un an après son $1^{\text {er }}$ examen en $1^{\text {re }}$ lecture à la Chambre des députés ${ }^{23}$. D'autres cas visent 3 lois qui ont été approuvées avec 7 lectures parlementaires en 1957,1967 et $1992^{24}$.

Ainsi, le Sénat italien n'a pas abusé de son rôle pour retarder excessivement l'approbation des lois. En raison de sa composition identique à la première chambre due à un scrutin électoral similaire, les majorités présentes aux deux chambres ont toujours coïncidé faisant de la deuxième chambre un doublon de la première, évitant par une composition différente entre la première et la deuxième la mise en place d'une cohabitation législative pouvant augmenter la possibilité de différends entre les deux chambres. La mise en place du bicamérisme prêt de la perfection ${ }^{25}$ est déterminée par la législation électorale qui, en régissant la discipline constitutionnelle de la procédure législative, constitue la loi organique du bicamérisme italien.

Les variations du bicamérisme sont intimement liées par le mode de scrutin.

$\mathrm{Si}$, constitutionnellement, les deux chambres ont les mêmes compétences, la législation électorale a la possibilité d'altérer l'homogénéité de composition existante entre les deux assemblées. C'est ce qui se produisit lors des élections de 2006 avec la loi 270/2005. En effet, l'introduction de cette législation électorale a romput la nature parfaitement égalitaire des deux chambres et mit en place un discrimen du bicamérisme : avant 2006, la nature égalitaire du bicamérisme prêt de la perfection était due à l'homogénéité électorale ; à partir de 2006 se mit en place un Sénat comme chambre de modération. Grâce au complexe mécanisme des bonus majoritaires prévus par la loi électorale, la majorité au Sénat put être réduite à la Chambre des députés, faisant du Sénat un barrage contre la tyrannie du bloc majoritaire présent à la Chambre des députés, et donc une chambre de ralentissement, voire d'enlisement. C'est ce qui se produisit lors des élections de 2006 où la gauche eut une majorité quasi inexistante (v. supra). La dite circonstance constitua un cas unique en raison de l'écart exigu entre majorité et opposition, mais laissa ouverte la possibilité à des majorités différentes entre les deux chambres.

La décision $n^{\circ}$ 1/2014 de la Cour Constitutionnelle déclarant inconstitutionnelle

23 Cf., (http://legislature.camera.it/frameset.asp?content=\%2Faltre\%5Fsezionism\%2F8793\%2F8 992\%2Fdocumentotesto\%2Easp\%3F).

24 Site internet de Legislature precedenti Camera dei deputati.

25 En ces termes, J. BOUDON et P. PASSAGLIA, "Studi Pisani sul Parlamento ", vol. VI, op. cit., p. 26, Pisa University Press, Octobre 2014. 
la loi électorale dans l'attribution du bonus majoritaire à l'échelle nationale et régionale (considérant en droit $n^{\circ} 3$ et 4$)^{26}$, mit fin à l'hétérogénéité électorale et reconduit le bicamérisme italien à son degré le plus pur de perfection. Une partie de la doctrine ${ }^{27}$ considérant la difficulté du législateur constitutionnel italien à réviser le bicamérisme, a envisagé la possibilité d'intervenir au travers de la législation ordinaire (loi électorale) et ensuite par les règlements parlementaires, pour mettre en place une diversité de fonctions entre le Sénat et la Chambre des députés et résoudre le problème majeur du bicamérisme italien : le chevauchement des deux chambres en raison de l'homogénéité des rôles. Une telle position mettait en place une réforme transitoire du bicamérisme, cachée sous l'apparence d'une réforme à la loi électorale, dans l'attente godotienne que soit enfin révisé le bicamérisme italien. À part l'utilisation à des fins abusives de la loi électorale pour agir dans des domaines réservés à l'initiative du pouvoir constituant dérivé, une telle possibilité doit être écartée en raison de la jurisprudence émise par la Cour Constitutionnelle, ci-après CCI (décisions 1 de 2014, 35 de 2017). Déjà dans sa décision de 2014, la CCl, en censurant la loi électorale de l'époque, affirmait que celle-ci pouvait mettre en place des majorités hétérogènes, portant ainsi atteinte au fonctionnement de la forme de gouvernement parlementaire qui se concrétisait par la confiance dont avait bénéficié l'exécutif à l'égard des assemblées (art. 94) et par l'exercice paritaire de la fonction législative (art. 70). Enfin, dans la décision 35 de 2017 (considérant en droit $n^{\circ}$ 15.2) elle affirmait que si la Constitution n'obligeait pas le législateur à adopter des systèmes électoraux identiques, elle exigeait que ces derniers, pour ne pas compromettre le bon fonctionnement de la forme de gouvernement parlementaire, n'empêchent pas la formation de majorités parlementaires homogènes. Ainsi, si la CCI n'excluait pas la possibilité de mettre en place un certain degré d'hétérogénéité dans les scrutins électoraux en raison du contenu des normes constitutionnelles (élection sur base régionale pour le Sénat, art. 57, al. 1 constitution), il appartenait au législateur de modérer l'intensité de cette hétérogénéité afin de ne pas compromettre le bon fonctionnement du Parlement.

Pour la majorité des lois adoptées, on constate comment la seconde lecture effectuée par le Sénat a été la définitive. Cette tendance s'est confirmée aussi dans les législatures récentes, notamment les $\mathrm{XV}, \mathrm{XV} \mathrm{I}^{\mathrm{e}}$ et $\mathrm{XVIle}$ législatures, sous le nom de monocamérisme ${ }^{28}$ de fait ${ }^{29}$ en indiquant une procédure où il y a une

26 Cf., (http://www.giurcost.org/decisioni/2014/0001s-14.html).

27 P. PASSAGLIA, dans "Studi Pisani sul Parlamento ", op. cit., p. 27.

28 Le Professeur Jean-Philippe Derosier parle aussi de Monocamérisme. Ce dernier affirme qu'àce jour le bicamérisme égalitaire n'existe plus. En effet selon cet auteur si les chambres avaient les mêmes fonction et légitimation, une des deux finirait par devenir la photocopie de l'autre ce qui " reviendrait à ne désigner qu'une seule chambre, puis à la couper en deux ". Cf., J. DEROSIER, "Le bicamérisme : un défi et un enjeu démocratique ", Petites Affiches, n 45, 4 mars 2015, art. cit., p. 2.

29 C'est le Premier Ministre Giuliano Amato à la Chambre des députés, lors de la séance du 21 février 2001, qui utilisa ce mot. 
chambre qui commence l'examen et qui l'élabore (chambre active) et l'autre qui se limite de confirmer par une sorte de vote ratification, la décision prise par l'autre assemblée ${ }^{30}$.

Au vu de cette situation, le bicamérisme qui se met en place s'apparente plus à un bicamérisme spécialisé ${ }^{31}$ car il n'y a pas de vraie collaboration entre les deux chambres qui se se réaliserait par un exercice collectif de la fonction législative correspondant à une succession des passages dans l'accomplissement de leurs tâches ${ }^{32}$ : l'approbation d'un texte commun.

Dans ce bicamérisme, dont le meilleur exemple est représenté par la Constitution consulaire de l'an VIII ou 1795, il y a une répartition des tâches entre les deux chambres pendant la procédure législative : une chambre est chargée d'une fonction de préparation et d'élaboration du texte (Conseil des Cinq-Cent, art. 76), l'autre (Conseil des Anciens, article 86) est chargée d'approuver ou de rejeter in toto le texte au moyen d'un vote bloqué sans possibilité d'exercer son droit d'amendement.

Le bicamérisme contemporain italien serait une évolution de ce genus, réalisant un bicamérisme spécialisé alterné : la chambre qui se limite à ratifier le texte élaboré par la première, pourrait être indistinctement le Sénat ou la Chambre des députés.

Telle solution est une conséquence du régime particulier des navettes italiennes sur lequel la constitution se limite à prévoir l'approbation d'un texte commun par les deux chambres.

II faut observer toutefois qu'à partir de 1996 on assiste à une évolution de la procédure législative en raison du recours par le gouvernement à des procédés lui permettant d'accélérer l'approbation des lois. II s'agit notamment de la question de confiance qui permet à l'exécutif d'avoir recours à une sorte de vote bloqué avec toutefois des contraintes juridiques : l'exécutif engage sa responsabilité sur le texte et devra démissionner en cas d'échec. C'est pourquoi il paraît plus opportun de parler de vote bloqué à l'italienne. Un tel instrument est devenu une pratique habituel lors de la procédure législative italienne qui est utilisée de manière conjointe au maxi-amendement, ce dernier permettant d'adopter une loi entière par un simple amendement dérogeant ainsi au principe du "vote article par article " énoncé à l'article 72, alinéa 1 de la Constitution.

En observant les cas les plus répandus de navettes parlementaires, 1 navette en fonction de 3 lectures parlementaires (sénat, chambre, sénat ou chambre, sénat, chambre, ci-après on utilisera $S$. pour Sénat et $C$. pour chambre des députés), dans le schéma C, S, C on constate que le Sénat a un rôle de chambre active car il amende le texte venant de la chambre des députés et le texte amendé

30 Cf., E. LONGO, " La legge precaria, Le trasformazioni della procedura legislativa nell'età del l'accelerazione ", Giappichelli Editore, Torino, op. cit., p. 200.

31 J. BOUDON, art. cit., p. 5.

32 Idem. 
est ensuite approuvé définitivement par la chambre des députés qui n'amende pas le texte (chambre passive). Tel rôle de chambre active n'est pas présent dans le schéma S, C, S où c'est le Sénat a contrario, qui exerce le rôle de chambre passive. Si, théoriquement, ce schéma pourrait représenter les circonstances où le Sénat agit de manière déterminante sur le texte qui sera voté définitivement, l'absence d'une répartition des lois en fonction de l'initiative législative ne permet pas de le placer dans une situation de primauté quant au texte qui sera approuvé.

Ainsi les circonstances dans lesquelles se mettent en place une logique C, S, C ou S, C, S dépendent exclusivement de la chambre qui va la première inscrire à l'ordre du jour un texte de loi. En effet, il y a une sorte de compétition entre les deux chambres pour examiner en premier lieu un texte, selon la pratique parlementaire exigeant que la commission de la chambre commençant la première l'examen acquiertuneprioritéparrapportàl'autrechambrequidevraits'abstenirdel'examen ${ }^{33}$. La compétition pour inscrire en premier lieu le texte à l'ordre du jour est une constante de la vie parlementaire qui pourrait trouver une logique en présence d'un bicamérisme accordant à la chambre de $1^{\text {er }}$ examen le dernier mot en cas de modification par la $2^{\mathrm{e}}$ chambre ${ }^{34}$.

Il faut enfin ajouter que la chambre passive qui se désaissit du droit d'amendement sur le texte voté dans la lecture précédente, est tenue de voter en bloc le texte préalablement voté en $2^{\mathrm{e}}$ lecture grâce au recours par l'exécutif à des instruments de rationalisation parlementaire non consacrés directement en constitution (question de confiance). Ce qui constitue une nouvelle forme de rationalisation contemporaine de la procédure législative qui est désormais devenue une pratique constante.

\section{Le Sénat sous la III République}

Dans le régime parlementaire de l'époque, on observe la tentative de mettre en place un équilibre des pouvoirs, mais ce dernier, par les règles non écrites qui s'instaureront, dégénéra en raison de l'utilisation faite par les hommes politiques du moment. La dite dégénérescence conduira vers un régime parlementaire moniste qui finit en régime d'assemblée.

Si formellement le bicamérisme existant de l'époque demeure égalitaire, les deux chambres diffèrent quant à leur nature élective. Une telle différence était aussi présente dans le système parlementaire italien de l'époque (v. supra, page 9) qui se différenciait par la modalité d'élection des sénateurs : nommés par le Roi

33 D. RAVENNA dans Studi Pisani sul Parlamento, Volume VI, Pisa University Press, op. cit., p. 15.

34 Ce type de bicamérisme est présent au Brésil. Cf., Servizi Studi del Senato, marzo 2014 $\mathrm{n}^{\circ} 118$, dossier, XVII legislatura, Le Camere Alte nei paesi extraeuropei, ufficio di ricerche sulla legislazione comparata e per le relazioni con il CERDP, cit., p. 43. II est intéressant d'observer que le législateur constitutionnel italien, qui a essayé maintes fois de réviser le bicamérisme italien, s'est toujours inspiré, dans les nombreuses révisions proposées, au modèle allemand avec quelques adaptations (absence d'une commission mixte paritaire), mais n'a jamais pris en compte un modèle comparable à celui présent au Brésil. 
en Italie, au suffrage universel indirect en France. La différence entre les deux chambres italiennes permit la mise en place d'un unformal Italian Parliament Act qui de facto annula la toute puissance du Sénat: possibilité de révoquer la confiance à l'exécutif et de modifier les textes prolongeant indéfiniment la durée de la procédure législative. La dite pratique ne fut pas appliquée dans le bicamérisme de la III alors que d'autres pratiques non prévues en constitution se mirent en place ${ }^{35}$, ce qui exposa l'exécutif à un double rapport avec les chambres, au niveau de la confiance et de la procédure législative, faisant de ces dernières le dominus inconditionné du régime en place.

L'instabilité en matière électorale fut aussi un élément qui engendra une instabilité des exécutifs. Mais, même en présence d'une législation électorale stable, cette dernière se serait avérée inefficace en raison de I'hétérogénéité électorale due au scrutin du Sénat constitutionnellement différent.

L'initiative et la confection des lois étaient exercées concurremment par les

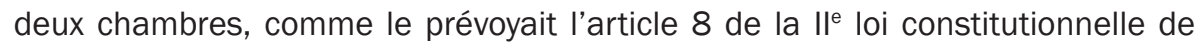
1875. Malgré la formule employée qui mettait en place un exercice paritaire de la deuxième chambre par rapport à la fonction législative, il y avait une volonté de coopération des deux chambres. Celle-ci était témoignée par les règlements parlementaires de l'époque qui mirent en place, sous l'influence du modèle parlementaire anglais et américain, des conférences de conciliation ${ }^{36}$.

A l'inverse de la Constitution du 4 octobre 1958, les lois constitutionnelles ne codifiaient pas dans la norme fondamentale la procédure de conciliation; ce fut lors de la rédaction des règlements parlementaires qu'une telle nécessité fut soulevée ${ }^{37}$, pour pallier aux lacunes de la norme fondamentale en la matière.

Les articles mettant en place la dite procédure étaient les articles 130 et 131 du règlement du Sénat ${ }^{38}$ et 140, 141, 142 du règlement de la Chambre des députés ${ }^{39}$. Ils étaient ainsi rédigés ${ }^{40}$ :

\section{Règlement du Sénat}

Art. 130 " Lorsqu'un projet de loi voté par le Sénat a été modifié par la Chambre des députés, le Sénat peut ou mettre de nouveau ce projet en délibération ou le sou-

35 La mise en place des décrets-lois s'expliquait comme un moyen palliatif, pour répondre à la nécessité et à l'urgence, remédiant ainsi aux lenteurs du Parlement. Un éventuel recours à une sorte de Paliament Act heurtait avec les prérogatives du Sénat en matière de dissolution de la chambre des députés. Néanmoins, la norme contenue dans l'article 5 de la lère loi constitutionnelle de 1875 est tombée en désuétude et s'est avérée inutilisable en raison de l'esprit de la Constitution de Grévy qui se traduisait par la non-utilisation du droit de dissolution. Intervention du Sénateur Clément, séance du 10 juin 1876, Annales du Sénat, session de 1876, p. 236, in fine.

37 M.-D. PIERRE, L'article 45 de la Constitution du 4 octobre 1958, Rationalisation de la navette parlementaire et équilibre des pouvoirs constitutionnels, LGDJ, op. cit., p. 45.

38 Le Sénat approuva ces articles avant la Chambre des députés le 10 juin 1876.

39 Articles votés par la Chambre des députés le 16 juin 1876.

40 M.-D. PIERRE, art. cit., p. 46. 
mettre aux bureaux, ou le renvoyer à l'ancienne commission. II peut également, sur la proposition d'un de ses membres, décider qu'une commission sera chargée de travailler avec une commission de la chambre des députés pour s'entendre sur un texte commun. Le Sénat donne les pouvoirs à cet effet à une commission de onze membres élus au scrutin de liste".

Art. 131 "Si les deux commissions tombent d'accord, la commission nommée par le Sénat fait un rapport à cette assemblée qui délibère sur la nouvelle rédaction. Si le Sénat a repoussé la proposition d'une conférence, le projet ne pourra être porté de nouveau à l'ordre du jour avant le délai de deux mois, que sur l'initiative du gouvernement. II en sera de même dans le cas où les commissions ne tomberaient pas d'accord, ou si le Sénat persistait dans sa première résolution ".

\section{Règlement de la Chambre des députés}

Art. 140 "Lorsqu'un projet de loi, voté par la Chambre des députés, a été modifié par le Sénat, la Chambre des députés peut décider, sur la proposition d'un de ses membres, qu'une commission sera chargée de se réunir avec une commission du Sénat, afin de s'entendre sur un texte commun. La Chambre des députés décidera si les pouvoirs, à cet effet, seront donnés à la commission qui a fait le rapport sur le projet, ou à une nouvelle commission élue dans les bureaux".

Art. 141 " Si les deux commissions s'entendent sur un texte, la commission nommée par la Chambre des députés fait un rapport à cette assemblée. La chambre des députés délibère sur la nouvelle rédaction ".

Art. 142 " Si la Chambre des députés a repoussé la proposition d'une conférence, le projet ne pourra être porté de nouveau à l'ordre du jour avant le délai de deux mois que sur l'initiative du gouvernement. II en sera de même dans le cas où les deux commissions ne s'entendront pas sur un texte ou si la Chambre persiste dans sa première résolution ".

En lisant ces articles, on constate que la procédure prévue n'est pas contraignante, comme en témoignent les formules utilisées aux articles 130 et 140 des deux règlements qui emploient le formule "peut décider ". En effet, Iorsqu'un texte est amendé par la chambre de deuxième examen, la chambre de premier examen dispose de la possibilité de convoquer une commission qui sera chargée d'entrer en contact avec la commission de l'autre assemblée.

Si la chambre des députés en présence d'un texte modifié par le Sénat, dispose seulement de la possibilité de convoquer dite commission, le Sénat pour sa part , a plusieurs options devant lui, ce qui constitue une hétérogénéité par rapport au règlement de la chambre des députés : demander une nouvelle délibération du texte, soumettre ce dernier à l'examen des bureaux (l'organe existant avant l'institution des commissions permanentes), le renvoyer à la commission chargée de l'examen. La possibilité de convoquer la commission est aussi prévue mais constitue une option parmi plusieurs choix alternatifs, comme en témoigne le 
début de la $2^{\mathrm{e}}$ phrase du $1^{\mathrm{er}}$ alinéa de l'article 130 " II peut également [...] ".

En outre, si le Sénat nomme une commission de 11 membres élus au scrutin de liste, le règlement de la Chambre des députés n'indique aucun effectif numérique mais prévoit, lors de la décision de convoquer la commission, de donner les pouvoirs à la commission qui a fait le rapport sur le projet ou à une nouvelle commission. Cette disposition, apparemment d'ordre technique permet d'observer que le système de conciliation sous la III ${ }^{e}$ République n'était pas nécessairement paritaire; le règlement de la chambre des députés ne prévoyait pas le nombre de membres de la commission, mais renvoyait le choix à la commission chargée du rapport ou à une autre commission : les commissions prévoyaient des effectifs différents en raison du nombre des députés supérieur à celui des sénateurs. Pour éviter un nombre différent, il fut prévu, le 11 décembre 1879 à la chambre des députés, que la nomination de cette commission, serait fixée à un membre pour chaque bureau, soit 11 commissaires en raison des 11 bureaux présents. Toutefois, la chambre des députés, le 7 juin 1889, adopta une décision contraire en confiant les pouvoirs de la commission de conciliation à la commission saisie au fond qui était plus nombreuse que celle présente au Sénat : 33 députés contre 18 sénateurs. Une problématique identique se posa à nouveau en $1909^{41}$.

On observe ainsi que les deux règlements prévoyaient deux procédures différentes pour choisir la commission qui était chargée de faire face aux divergences entre les deux chambres et asseoir un compromis.

Le système de conciliation établie par les deux règlements reposait sur la mise en place de deux organismes chargés de trouver un point d'accord sur les dispositions d'une loi en cours de discussion.

On dénombre 7 phases respectives dans la procédure.

La première phase est celle de l'initiative, à la demande d'un sénateur ou député au moyen d'une motion dans laquelle on charge la commission saisie au fond de la chambre de référence de se réunir avec une commission de l'autre assemblée pour trouver un compromis sur un texte en discussion.

La deuxième phase consiste au vote de la motion présentée.

Si cette motion est approuvée s'ouvre la troisième phase qui consiste à la transmission d'une lettre du Président de la chambre adoptant la motion dans laquelle il informe son homologue de la décision de convoquer une commission pour asseoir un compromis.

La quatrième phase consiste en la délibération de l'autre chambre sur l'opportunité d'entrer en lien avec la chambre qui a préalablement adopté la motion pour convoquer les commissions de conciliation. On observe ainsi que la procédure de convocation de ces deux commissions répond à l'esprit égalitaire du bicamérisme, prévoyant l'assentiment des deux chambres sans possibilité pour l'exécutif d'intervenir dans la procédure : en cas de vote négatif, la procédure

41 Ibidem, p. 50. 
s'arrête ${ }^{42}$.

En cas de vote positif par l'autre chambre, s'ouvre la cinquième phase qui consiste en la réunion conjointe des deux commissions en vue d'approuver un texte commun.

La sixième phase concerne le vote. En effet, si les deux commissions se réunissent conjointement pour élaborer un texte commun, elles se séparent au moment du vote pour délibérer séparément sur le rapport élaboré (on constate ici la similitude avec le système des Conférence Commitee aux U.S.A. et des Joint Commitee mis en place au Royaume-Uni avant que les Parliamentary Act de 1911 et 1949 ne consacrent une primauté à la chambre basse anglaise). Ainsi, le mécanisme retenu ne met pas en place une entité juridique ; il s'agit d'une réunion de deux commissions, chacune en fonction de représentation d'une des deux chambres. La nature non paritaire est témoignée, outre la question des effectifs numériques, par le fait que les deux commissions se séparent au moment du vote.

Cette sixième phase peut aboutir à un échec si le rapport n'est pas approuvé dans les mêmes termes.

En cas d'approbation identique s'ouvre la septième et dernière étape qui consiste à l'envoi du texte au Président de la République pour promulgation.

Cette procédure de conciliation, qui témoigne de la volonté des assemblées à mettre en place une forme de rationalisation de la procédure législative, s'avéra dans les faits inefficace. Seules trois commissions de conciliation se réunirent Iors de la III République : en 1880 pour la loi sur la réorganisation de l'Étatmajor, en 1889 pour la loi relative au recrutement, et enfin en 1909 pour la loi relative à l'utilisation de la céruse dans les travaux peintures exécutés tant à l'extérieur qu' à l'intérieur ${ }^{43}$.

L'échec de ce mécanisme s'expliquait en raison du fait que les dites commissions agissaient comme deux corps parallèles, ce qui rendait difficile de dégager un accord commun entre ces organes.

En outre, il fallait observer que la procédure de convocation ne demeure pas une procédure de droit commun, activable immédiatement à partir du moment où une des deux chambres amendait le texte voté par l'autre, mais était un procédé exceptionnel et éventuel dont l'utilisation était laissée à un jugement d'opportunité des deux chambres.

L'échec des commissions de conciliation témoigne du double pouvoir dont bénéficiait le Sénat lors de la procédure législative : comme ce dernier était sur un pied d'égalité avec la chambre des députés, il était en mesure de refuser la procédure de conciliation ou de rejeter son rapport. II était ainsi le dominus de la procédure législative, en raison des dispositions réglementaires des députés qui

42 Ibidem, p. 49.

43 Ibidem, p. 52. 
n'accordaient pas à ces derniers le dernier mot, et des prérogatives qui lui étaient accordées constitutionnellement. La procédure de conciliation mise en place était conçue pour essayer de remédier aux problèmes du bicamérisme égalitaire de l'époque, mais en donnant au Sénat les mêmes prérogatives, elles finirent par être inefficaces.

\section{La singularité du bicamérisme italien}

Si l'Italie ne constitue pas un cas isolé de bicamérisme égalitaire, d'autres pays ont un Parlement où les deux chambres exercent sur un pied d'égalité les mêmes compétences lors de la procédure législative (A), l'octroi de la double confiance constitue un des éléments les plus caractéristiques du Parlement italien (B).

\section{A. Les bicamérismes égalitaires ou semi-égalitaires contemporains}

L'exercice des pouvoirs identiques dans la procédure législative se retrouve dans d'autres Parlements. C'est le cas en Europe ${ }^{44}$, de la Suisse, de la BosnieHerzégovine et des Pays-Bas (1), et des États-Unis et l'Australie dans les pays extra-européens (2).

\section{L'État présent en Europe}

Si on considère les deux structures des Parlements répandues aujourd'hui, on observe qu'en Europe 31 parlements sont monocamérales alors que le bicamérisme est adopté dans 17 pays, dont 4 sont égalitaires. Ces chiffres extraits par les données de I'Union Interparlementaire soulignent une primauté du monocamérisme sur le bicamérisme. Toutefois, on peut parler d'autre part d'une primauté cachée du bicamérisme sur le monocamérisme, dans une logique qualitative et non plus quantitative. En effet, si on analyse en détail les pays qui adoptent le bicamérisme, on observe que tous les pays du G8 sont bicaméraux, 15 pays du G20 sont bicaméraux. C'est ainsi que 4 milliards de personnes (si on exclut la Chine) sont représentés par des systèmes bicaméraux : 30 des 40 pays les plus peuplés au monde sont bicaméraux. Si on traduit ces données en superficie géographique, celle des pays ayant une structure bicamérale est beaucoup plus étendue que celle des pays monocaméraux ${ }^{45}$.

Si le choix du type de Parlement repose sur deux variantes, il est difficile de fournir une théorie générale solide et sans équivoque quant au système adopté

44 On prend en compte les pays faisant partie géographiquement de l'Europe et non ceux appartenant uniquement à l'Union Européenne.

45 Dossier ${ }^{\circ} 54$, Servizio studi del Senato, art. cit., p. 9. 
car il n'existe pas de lien direct entre un modèle et les caractéristiques du pays concerné.

Un Pays (Bosnie-Herzégovine) met en place un bicamérisme égalitaire singulier dans le cadre d'une forme de gouvernement similaire pour la Bosnie-Herzégovine (forme de gouvernement parlementaire fédérale) (b).

Avant la révision de 2003, la Roumanie, qui adopte une forme de gouvernement semi-présidentielle à l'image du modèle français ${ }^{46}$ avec des procédés qui s'apparentent au parlementarisme rationalisé, avait un bicamérisme égalitaire indifférencié à l'instar du modèle italien. La réforme de 2003 a introduit un bicamérisme inégalitaire concernant la procédure législative qui s'apparente d'une certaine manière au bicamérisme inégalitaire existant en Allemagne, en raison de la répartition des tâches entre les deux chambres grâce à une liste de matières.

Les Pays-bas pour leur part constituent le modèle qui se rapproche le plus du système italien en raison de la similitude avec la forme de gouvernement existant en Italie (a).

La Suisse a un bicamérisme égalitaire inséré dans une forme de gouvernement directoriale différente de la forme parlementaire (c).

\section{a. Le bicamérisme Hollandais}

Le Parlement des Pays-bas (Staten-Generaal) se compose de deux chambres qui prennent le nom de Eesrte Kamer (première chambre) et la Tweed Kamer (seconde chambre).

La Eerst Kamer correspond à la chambre haute, alors que la Tweed Kamer est la chambre basse. Ainsi on constate que malgré le terme utilisé (première chambre pour la Erste Kamer, et seconde chambre Tweed Kamer) la première chambre n'est pas dans une position de primauté par rapport à la seconde chambre. Le

46 La Constitution de la Roumanie peut être définie à juste titre la plus francophile des constitutions européennes. En effet, son article 113 met en place un mécanisme quasi similaire à celui représenté par l'engagement de responsabilité de l'article 49 alinéa 3 de la Constitution. Cet article prévoit "(1) le Gouvernement peut engager sa responsabilité devant la chambre des députés et le Sénat, en séance commune, sur son programme, une déclaration de politique générale ou un projet de loi. (2) Le Gouvernement est démis si une motion de censure, déposée dans les 3 jours, est approuvée à compter de la présentation du programme, de la déclaration politique générale ou du projet de loi, a été votée dans les conditions fixées à l'article 112. (3) Si le Gouvernement n'a pas été démis conformément à l'alinéa 2, le projet de loi était considéré comme adopté [...] ". On constate ainsi la proximité de ce procédé à celui du 49.3 en France avec des variantes qui sont constituées en premier lieu par le vote en séance commune du Parlement. Deuxièmement, par la fusion du mécanisme prévu par le 49.3 et l'engagement de responsabilité prévu par l'art. 49 1. F. VASILESCU, "Considérations sur le régime politique actuel en Roumanie ", Rev. Dr. Const., 1995, n²3, op. cit., p. 541. Néanmoins par rapport aux deux éléments qui distinguent le 49.3 français de l'article roumain, il convient de préciser que la Constitution roumaine prévoit aussi la possibilité d'un réexamen, car le président de la République a la faculté de demander un réexamen de la loi adoptée en vertu du procédé prévu à l'alinéa 3. C'est ainsi que cette possibilité d'examen peut constituer une sorte de check and balances par rapport à une procédure déjà rationalisée. 
terme employé est sans répercussion sur l'exercice de ses attributions.

La Erste kamer se compose de 75 membres élus au suffrage indirect par les Conseils législatifs de 12 provinces qui composent le pays pour un mandat de 4 ans.

La Tweed Kamer est formée par 150 membres élus au suffrage universel direct pour un mandat de 4 ans au scrutin proportionnel.

La Constitution attribue formellement les mêmes compétences aux deux chambres dotées du pouvoir législatif, d'enquête, budgétaire et de détermination de la ligne politique ${ }^{47}$.

Cas singulier, la Constitution hollandaise ne contient aucune disposition sur la responsabilité politique de l'exécutif face au Parlement. La Constitution se limite à prévoir (art. 65) que l'exécutif présente chaque année (le troisième mardi de septembre) devant les États-Généraux, une déclaration de politique générale.

L'absence de responsabilité dans la constitution matérielle pourrait laisser penser à une irresponsabilité de l'exécutif. En réalité, même en l'absence de règles écrites, la responsabilité de l'exécutif devant le Parlement s'est mise en place par des conventions constitutionnelles. Comme l'a relevé une part de la doctrine, en l'absence de dispositions constitutionnelles sur la confiance parlementaire, la pratique parlementaire permet au législatif de voter des motions (moties) qui matériellement, ont une portée équivalente à la motion de défiance, ou de voter d'autres actes législatifs finalisés pour provoquer la démission d'un ministre, comme par exemple la réduction de son salaire à un seuil symbolique (50 centimes) $^{48}$. Ce qui constitue vraiment un cas sui generis car la Constitution demeure lacunaire quant à la responsabilité de l'exécutif ; mais en l'absence d'une telle discipline, on parvient par des règles non écrites à mettre en place non seulement la responsabilité de l'exécutif devant le Parlement, mais aussi la responsabilité individuelle des Ministres.

S'agissant de la fonction législative, la Eerste Kamer n'a ni l'initiative législative ni la possibilité d'amender les textes venants de la Tweed Kamer. II y a ainsi une plénitude législative de la Tweed Kamer alors que la Erste Kamern'a pas le pouvoir d'amender les textes mais seulement de les approuver ou de les rejeter " tels qu'ils lui ont été envoyés par la Eerste Kamer" (art. 85, $1^{\text {er }}$ alinéa de la constitution ${ }^{49}$ ). Se référant à ce type de bicamérisme lors de la procédure législative on peut parler de bicamérisme symétrique non paritaire ${ }^{50}$ qui correspond au modèle de bicamérisme spécialisé de Julien Boudon (v. supra p. 10). L'impossibilité pour la Eerste Kamer de pouvoir exercer son droit d'amendement entrave ses

47 Dossier $n^{\circ}$ 54, Ibidem, op. cit., p. 83.

48 R.-D. ANDEWEB, G.-A. IRWIN, Governance and politics of the Netherlands, Palgrave MacMillan, Houndmills-New York, Ivédition, 2014, op. cit., p. 173.

49 Digithèque de matériaux juridiques (ci-après Digithèque MJP) et politiques de I'Université de Perpignan.

50 A. LIJHPART, Patterns of democracy: Government Forms and performance in Thirty-Six Countries, Yale University Press, New Heaven-London, 2012, op. cit., p. 193. 
prérogatives égalitaires par rapport à la Tweed Kamer.

La constitution hollandaise met en place de iure un bicamérisme dont le fonctionnement au niveau de la procédure législative est comparable à celui de la constitution de I'an VIII (1795). II s'agit ainsi d'un exemple contemporain d'un ancien modèle de bicamérisme qui, à ce jour, n'existe plus. Si le bicamérisme italien de facto s'apparente à ce type de bicamérisme, la spécificité du cas hollandais repose sur sa discipline constitutionnelle. Le bicamérisme italien qui est de facto spécialisé demeure de iure parfaitement égalitaire. C'est grâce aux effets implicites de la rationalisation parlementaire (question de confiance) que ce dernier perd sa nature parfaitement égalitaire.

De iure, la Eerste Kamer peut approuver ou rejeter in toto les textes transmis par la Tweed Kamer, cette dernière a la possibilité de jouer un rôle d'avocat concernant le texte qu'elle approuve car elle peut nommer un ou plusieurs membres qui auront la tâche de défendre le texte en cours d'examen à la Tweed Kamer (article 85, 2e alinéa) ${ }^{51}$. Toutefois, malgré l'impossibilité d'exercer un pouvoir d'amendement, de facto les débats parlementaires qui ont lieu dans cette chambre contribuent à éclairer l'interprétation des textes en examen à l'avantage de la relative jurisprudence. L'absence d'amender le texte n'empêche pas à la première chambre de le rejeter, exerçant ainsi un veto définitif qui entraîne la fin de la procédure législative : suite à un rejet, la navette ne peut se poursuivre et aucune commission de conciliation ne peut être convoquée ${ }^{52}$. C'est ainsi que pour éviter un potentiel vote négatif de la Eerste Kamer, le gouvernement prend en compte les avis formulés par cette dernière. Dans la pratique, le gouvernement pour asseoir un compromis finalisé et obtenir l'avis favorable du Sénat, présente un projet de loi supplémentaire qui prend en compte les observations faites par la Eerste Kamer.

Si de iure le bicamérisme hollandais est un bicamérisme spécialisé classique, de facto la pratique met en place un bicamérisme égalitaire de collaboration en raison de la prise en compte des avis venant de la première chambre.

\section{b. Le bicamérismes égalitaire singulier de la Bosnie-Herzégovine}

Le système parlementaire mis en place en Bosnie-Herzégovine est le produit du droit international public, notamment les Accords des Dayton de 1995 (General Framework Agreement for peace - GFAP) après le conflit de 1992-1995 entre les différentes ethnies du pays (croates, bosniaques- musulmans, serbes). Notamment l'article 4 de cet accord qui fait un renvoi explicite aux compromis qui ont été atteints dans l'annexe 4 contenant la Constitution de 1995 de BosnieHerzégovine, imposée de l'extérieur aux belligérants. Une telle constitution met en

51 Digithèque MJP.

52 Si aucune conciliation n'est mise en place, le mécanisme prévu par l'art. 85, 2e alinéa (v. supra) s'apparente à une logique finalisée à trouver un compromis de manière indirecte par le rôle d'avocat défenseur que joue la Tweed Kamer. 
place dans le pays deux macro-régions, définies comme Entités (art. 1, alinéa 3) : la Fédération de Bosnie-Herzégovine $(\mathrm{FBIH})$, croate-musulmane $(51 \%$ du territoire), la République Srpska (RS) serbe (49\% du territoire).

À compter de 2009 a été approuvée une révision de la constitution (amendement $n^{\circ} 1$ de 2009) introduisant le District de Brcko qui constitue une collectivité autonome et s'ajoute aux deux autres entités existantes. La Constitution formalise les divisions territoriales qui avaient été conclues entre les parties et la finalité de garantir la sécurité entre les différentes ethnies, œuvrant ainsi comme loi organique des Accords de 1995. C'est ainsi que l'organisation institutionnelle se caractérise par un haut degré de décentralisation et par une division ethnique des institutions étatiques centrales. En effet, selon l'esprit de la Constitution c'est les deux entités qui confèrent légitimité à l'État et non l'inverse.

Le Parlement de la Bosnie-Herzégovine (Assemblée parlementaire) est composé par la Chambre des représentants et la Chambre des peuples.

La dite Chambre des représentants (Presdstavnicki Dom) se compose de 42 membres élus tous les 4 ans au suffrage universel par chaque entité au scrutin proportionnel : 28 membres sont élus par la Fbih et 13 par la Rs.

La dite chambre a pour fonction de représenter en son sein la répartition du pays dans les deux entités en prenant en compte le critère géographique qui accorde la majorité du territoire à la Fbih. C'est ainsi qu'elle repropose sur le plan institutionnel la dimension nationale du pays, à l'instar de ce que fait la première chambre dans les pays fédéraux.

La Chambre des peuples (Dom Naroda Parlamenta Fedeacije Bosne i Hercegovine) se compose de 15 membres : 10 représentants de la FBIH (avec un nombre égalitaire entre Bosniaques et Croates) et 5 de la RS pour une durée de 4 ans. La composition de la chambre des peuples, qui prévoit une représentation égalitaire de chaque ethnie, a un impact significatif au niveau de la composition. Les membres de la chambre des représentants s'organisent en groupes politiques, alors que les membres de la chambre des peuples prévoient des groupes politiques sur base ethnique ${ }^{53}$ qui se sous-structurent autour des 3 groupes bosniaque, croate, serbe.

Les 10 représentants de la FBIH sont nommés par la Chambre des peuples de cette région, à l'instar des 5 représentants de la RS nommés par son Assemblée Nationale.

On constate la présence d'un monocamérisme sub-national en raison de la présence d'un Parlement des deux macros-régions qui composent le pays. Le Parlement de ces deux Entités joue un rôle important car il va nommer les membres qui vont siéger dans la Chambre des peuples de Bosnie-Herzégovine. Pour pouvoir être élu à la chambre des peuples, il est nécessaire d'avoir 18 ans.

La chambre des peuples exerce une fonction législative mais aussi une fonction de préservation des équilibres entre les entités qui composent le pays. C'est pour

53 (http://www.parlament.ba/Delegate/List?page=1\&mandateld=10\&memberType=1). 
répondre à cette exigence que la Constitution prévoit un fonctionnement singulier de cette chambre, à partir d'un quorum calculé sur la base des différentes ethnies. En effet, le quorum de la chambre des peuples est de 9 membres, à condition que 3 bosniaques, 3 serbes et 3 croates soient présents (article IV, alinéa 1, lettre b de la Constitution).

La procédure législative demeure égalitaire comme le prévoit sans équivoque l'art. IV, alinéa 3, lettre c). Néanmoins, cette dernière (art. IV, alinéa 3, lettre d)) prévoit différentes étapes et un système de quorum très complexe malgré l'apparente simple disposition "Toute décision dans les deux chambres est prise à la majorité des députés présents et participant au vote " (première phrase de la lettre d) de l'alinéa 3). Pour que les délibérations soient valables, il est nécessaire que soit réuni $1 / 3$ des votes des membres appartenants à chaque entité du Pays. Si ce seuil n'est pas atteint, est convoquée une commission chargée d'asseoir un compromis et d'approuver le texte dans les 3 jours. En cas d'échec, un nouveau scrutin est organisé où est requise la majorité simple, mais l'adoption du texte est valable uniquement si les votes contraires ne comprennent pas $2 / 3$ ou plus des membres d'une entité.

Après avoir été adopté par la Chambre des représentants, le texte est transmis à la Chambre des peuples où il est examiné en suivant la même procédure qui se distingue seulement pour la particularité du quorum requis pour que les séances puissent avoir lieu (v. supra). En cas de modification du texte est prévue la réunion de la commission mixte pour l'harmonisation formée par 6 membres, 3 membres pour chaque chambre. Le texte élaboré est ensuite envoyé aux deux chambres pour y être approuvé, à défaut d'approbation par une seule chambre le texte est considéré comme définitivement rejeté. Cette procédure de conciliation s'applique aussi en cas de révision constitutionnelle laquelle se déroule en revanche par une réunion du Parlement en séance conjointe délibérant à la majorité de 2/3 des présents.

La Chambre des peuples exerce aussi un pouvoir d'équilibres des entités. En effet, une proposition de loi peut être déclarée contraire aux intérêts vitaux des entités ${ }^{54}$ (art. IV alinéa 3, lettre e)) bosniaques, croates et serbes par un vote exprimé à la majorité des membres de l'ethnie de référence. Dans cette circonstance, le Président de la chambre des peuples convoque une commission spéciale qui se compose d'un membre représentant chaque ethnie, chargé de trouver une solution. En cas d'échec, dans les 5 jours suivants ${ }^{55}$, la question est

54 La Cour Constitutionnelle par sa jurisprudence en la matière est très limitée (deux décisions, U-2/04, U-2/08). Ainsi, a émergé un catalogue d'intérêts vitaux en incluant : la dignité humaine, la liberté religieuse, le respect de l'identité culturelle et des identités présentes dans le pays, la confiance dans le social et dans les institutions politiques qui mettent en œuvre la participation sociale (décision U-2/04, VI Merits, paragraphe 31). D'autre part, la Cour a dégagé aussi un principe plus général, à propos de la protection des intérêts vitaux précisant que ces derniers doivent garantir la protection des droits individuels et des droits des groupes minoritaires. 
soumise par le Président de la Chambre des peuples ${ }^{56}$ à la Cour Constitutionnelle qui se prononce en la matière ${ }^{57}$, entraînant une suspension du débat parlementaire en cours ${ }^{58}$. Ce type de procédé constitue un cas particulier de contrôle a priori59 sur une proposition de loi visant le respect des intérêts des entités qui composent le pays.

Si la Bosnie-Herzégovine est souvent citée comme un des pays ayant un bicamérisme égalitaire ${ }^{60}$, on constate comment ce dernier constitue vraiment un cas ad unicum en raison de sa composition et structuration. Pour cette raison il paraît opportun de parler de bicamérisme ethnique égalitaire. Néanmoins, la présence du pouvoir dont dispose la Chambre des peuples qui constitue un contrôle avec effet suspensif sur le débat parlementaire en cours, attribue à cette chambre un pouvoir de garantie constitutionnelle propre et asymétrique par rapport au respect des intérêts vitaux. C'est ainsi que de iure il est difficile et réductif de parler simplement de bicamérisme égalitaire se référant au Parlement de ce pays. II conviendrait alors parler de bicamérisme ethnique égalitaire asymétrique.

\section{c. Le bicamérisme suisse}

En appliquant le principe du bicamérisme fédéral, l'Assemblée Fédérale se compose de deux chambres ou sections : le Conseil National, le Conseil des États. Toutefois, s'il y a une structure parlementaire fédérale, la nature de l'Assemblée reflète une volonté d'équilibre des intérêts des Cantons, afin d'éviter que les plus petits ne soient subordonnés aux plus grands.

Le Conseil National se compose de 200 députés élus au suffrage direct pour un mandat de 4 ans au scrutin proportionnel qui est constitutionnalisé (art. 149, alinéa 2 de la Constitution), échappant ainsi à la discrétion du législateur ordinaire.

Le Conseil des États, composé de 46 membres, a une fonction de représentation des cantons : 2 membres par canton, à l'exception de six cantons qui ont un membre par canton (art. 150, alinéas 1 et 2). Contrairement au Conseil National, la législation électorale est renvoyée au droit cantonal (art. 150, dernier alinéa). C'est ainsi que le droit cantonal pourrait envisager une législature différente par rapport à celle du Conseil National. Toutefois, tous les cantons ont décidé d'adopter une durée égale de 4 ans, alignant ainsi leur législature à celle du Conseil National. Ce souci d'alignement de la législature s'est traduit aussi dans

56 Article $16 \S 2$ of the Rules of Procedure of the Constitutional. (http://www.ustavnisud.ba/osnovni-akti/pravila-suda/drugi-dio/?title=uvod\&submenu=true).

57 Dossier n54, op. cit., pp. 41, 42, 45.

58 Décision U 2-04, Idem.

59 L'utilisation du mot a priori s'avère inexacte d'un point de vue formel car le contrôle n'intervient pas sur une loi approuvée définitivement dans l'attente d'être promulguée mais sur une loi encore à son état embryonnaire car celle-ci est encore à l'examen du Parlement. 
la majorité des cantons où les élections pour le renouvellement de la deuxième chambre de l'Assemblée Fédérale se tiennent le même jour que les élections du Conseil National, la seule exception concernant les cantons de Zoug et Appenzell Rodi qui prévoient un calendrier électoral différent ${ }^{61}$. L'alignement est présent aussi en matière électorale car les cantons ont adopté le scrutin majoritaire, à l'exception des deux cantons du Giura et Neuchâtel qui emploient le scrutin proportionnel.

S'il subsiste entre les deux chambres une durée quasi-homogène, les différences subsistant concernent la nature des chambres, l'une représentant la population de la Confédération, l'autre la population des Cantons. Néanmoins, cette différence n'impacte pas les fonctions des deux chambres qui ont les mêmes prérogatives lors de la procédure législative et de contrôle : bicamérisme égalitaire.

Les projets de loi examinés par une chambre sont immédiatement transmis à l'autre en une approbation identique, comme le prévoit la Constitution qui stipule : "les décisions de l'Assemblée Fédérale requièrent l'approbation des deux conseils " (article 156, alinéa 2). C'est la loi fédérale sur l'Assemblée Fédérale 171.10 qui constitue une véritable loi organique sur le fonctionnement et la procédure parlementaire. Son article 83, alinéa 1 repropose quasiment le même contenu de la disposition contenue en constitution à l'article 156 alinéa 2 "les décisions de l'Assemblée fédérale requièrent une concordance entre les deux chambres ". De même, l'article 89 alinéa 1 propose la logique traditionnelle des navettes parlementaires "si après la délibération d'un texte subsistent des divergences entre les deux chambres, dites décisions qui ne font pas l'objet d'un accord repartent à l'autre chambre jusqu'à ce qu'on parvienne à un accord " et celle de l' entonnoir " après la première délibération dans chacune des deux chambres, les délibérations successives concernent les dispositions qui n'ont pas l'objet d'un accord entre les chambres " (article 89, alinéa ${ }^{62}$ ).

C'est l'article 91, alinéa 1 qui prévoit une limite à ces régimes de navettes, prévoyant que si après 3 délibérations consécutives par chaque assemblée subsistent encore des divergences, on procède à la convocation d'une conférence de conciliation, composée de 26 membres à la hauteur de 13 membres pour chaque assemblée, chargée de trouver un accord (article 91 alinéas 1 et 2) ) $^{63}$. Cette conférence requiert la majorité des membres de chaque délibération et délibère à la majorité des membres. En cas d'égalité, le vote du Président est déterminant (article 92, alinéas 1 et 2) ${ }^{64}$. Ensuite, le texte est transmis aux assemblées pour son approbation. Cette conférence de conciliation s'apparente à la CMP française. Non seulement elle prévoit un nombre égal de membres mais

61 Dossier n54, op. cit., p. 154.

62 (https://www.parlament.ch/centers/documents/it/handbuch-bundesversammlung-i.pdf).

63 Ibidem

64 Ibidem. 
elle agit comme un organe unitaire en délibérant en réunion commune sans que les deux délégations se séparent au moment du vote, à l'instar du Conference Commitee américain. En outre, on constate que sa convocation est de droit après 3 délibérations infructueuses par les deux chambres.

\section{L'Etat présent dans les pays extra-européens}

Hors du continent européen, on dénombre 2 cas de bicamérisme égalitaire ${ }^{65}$. Parmi ces pays figurait aussi la Colombie ${ }^{66}$ avant la révision de 2015 qui a modifié la nature du bicamérisme.

\section{a. Le bicamérisme américain}

En raison de l'attribution de ces compétences identiques lors de la procédure législative mais surtout de compétences propres (approbation de traités conclus par le président à la majorité de $2 / 3$, nomination des hauts fonctionnaires fédéraux et des juges à la Cour suprême) et de l'adoption du $17^{\mathrm{e}}$ amendement ratifié en 1913 qui a instauré l'élection au suffrage universel des sénateurs par les législatures des États, le Sénat américain a une autorité majeure que celle de

65 Concernant le Chili on ne peut pas parler de bicamérisme égalitaire car les deux chambres ne sont pas parfaitement positionnées sur le même pied d'égalité lors de la procédure législative. En effet, dans ce contexte on a une procédure législative avec intervention présidentielle. Dans un premier cas (art. 69), il est prévu qu'en cas de rejet d'une loi par une des deux chambres du Parlement (Chambre des députés, Sénat) la chambre de rejet ne pourra réexaminer le texte avant un an. Néanmoins, le président de la République a la possibilité de mettre fin à ce délai suspensif, lorsqu'il s'agit d'une loi initiative présidentielle, en demandant à l'autre chambre de se prononcer à la majorité de 2/3. Dans ces circonstances, la loi sera à nouveau examinée par la première chambre et sera considérée comme rejetée seulement par un vote à la majorité des $2 / 3$. En ce qui concerne la procédure législative, sont prévues deux possibilités. La première (art. 70) vise la situation où la deuxième chambre rejette in toto un texte approuvé par la première. C'est alors que ce dernier sera examiné par une CMP et le texte sera définitivement approuvé en cas d'approbation identique par les deux chambres. Lorsque la Chambre de premier examen rejette le texte de la CMP, le président de la République peut demander à cette dernière de manifester sa volonté de rejet par un vote à la majorité des $2 / 3$ des présents. Si cela se produit, la chambre de premier examen se prononcera définitivement par un vote à la majorité des $2 / 3$ et lorsque ce quorum est atteint, le texte sera considéré comme définitivement rejeté. La deuxième situation se produit lorsqu'une chambre apporte des amendements à un texte et ces derniers ne sont pas approuvés par la chambre de premier examen (art. 71). Dans cette hypothèse, on procède à la convocation de la CMP. En cas d'echec, le Président de la République peut solliciter la chambre de première lecture à réexaminer le texte modifié par la deuxième chambre. En cas de rejet du texte à la majorité des $2 / 3$ des présents par la première chambre, ce dernier resta considéré définitivement rejeté. Toutefois, si la chambre de premier examen n'atteint pas les 2/3 (article 71 , alinéa 2), le texte sera examiné par la deuxième chambre et si cette dernière approuve le texte à la majorité des $2 / 3$, le texte sera considéré comme approuvé. Cf. (https://www.constituteproject.org/constitution/Chile_2015?lang=en). On constate donc que le bicamérisme ne demeure pas pleinement égalitaire puisque le quorum des 2/3 atteint par la deuxième chambre assume la valeur d'un dernier mot renforcé, ce qui s'apparente à une logique d'un bicamérisme inégalitaire.

66 A. LIJPHART, Patterns of Democracy. Governement Forms and performance in thiry-six Countries, New Heaven, London, Yale University Press, 1999, op. cit., p. 224. 
la Chambre des représentants.

Si le Sénat américain partage avec le Sénat italien l'élection au suffrage direct, son autorité accrue est déterminée par des prérogatives propres qui font de cette chambre un interlocuteur obligé avec le pouvoir exécutif67. Cette nature d'interlocuteur est rendue possible par une autre disposition constitutionnelle, notamment la durée du mandat des sénateurs, différente de celle des représentants.

L'attribution de mêmes compétences lors de la procédure législative entraîne une procédure législative similaire à celle présente dans d'autres pays (Suisse, Bosnie-Herzégovine) où on essaie d'asseoir un compromis par la réunion d'un organe de conciliation entre les deux chambres, faute de quoi la procédure législative se termine avec un texte qui n'est pas adopté.

En plus des compétences accordées par la Constitution, le rôle important du Sénat ne peut se comprendre qu'à travers une intégration par son règlement parlementaire qui accorde des pouvoirs significatifs aux sénateurs.

On parle d'une place centrale se référant à ce dernier. En effet, si la Constitution de sa part (article 1, section 5) prévoit que " chaque chambre pourra établir son règlement intérieur ", ce dernier peut être adopté et modifié à une majorité simple. Ce qui pourrait laisser penser à un droit parlementaire vivant en raison de l'absence de quorum aggravé. Dans les faits, une majorité simple est suffisante, mais en cas d'obstructionnisme il est nécessaire d'approuver une motion de clôture à la majorité des $3 / 5$ des sénateurs, faute de quoi il ne sera pas possible de mettre fin au filibustering. Ce qui explique dans les faits les nombreux échecs pour réviser ce règlement qui, apparemment, demeure simple à réviser mais qui devient impossible en raison du filibustering, notamment l'utilisation abusive du droit de parole (Rules XIX) qui met en place un droit parlementaire codifié stagnant.

En lisant le règlement du Sénat américain (Rules of the Senate) qui a fait l'objet d'une révision le 24 janvier 2013, on constate la volonté dans le droit parlementaire américain de vouloir préserver le droit d'expression des sénateurs avec un souci d 'efficacité qui s'est imposé progressivement et avec modération. La volonté de préserver les droits des sénateurs se met en place par le principe de la liberté de parole qui est utilisé à des fins dilatoires pour retarder l'approbation d'une loi (bill). Ainsi, le filibustering permet d'éviter de retarder le vote d'un texte aussi longtemps que le sénateur exerce son droit de parole. Comme on l'a observé ${ }^{68}$, jusqu'en 1917 aucun moyen de procédure ne pouvait être mis en place pour clôturer un débat et parvenir au vote final du texte. C'est à partir de cette année-là que s'est mise en place une rationalisation des débats sénatoriaux à l'initiative du Président Woodrow Wilson par l'introduction de la Rule XXII qui

67 P. LAVAUX, A. LE DIVELLEC, Les grandes démocraties contemporaines, $4^{e}$ Éd, Collection de droit fondamental, Puf, op. cit., p. 304. 
constitue la traduction au Congrès américain de la guillotine motion au Parlement anglais. La règle introduite en 1917 permet l'approbation d'une motion to close the debate avec le même quorum requis pour œuvrer l'impeachement du Président des États-Unis : les 2/3, soit 66 sénateurs. Cette règle fit à son tour l'objet d'une modification en 1975, qui consista en un abaissement du quorum au 3/5 des sénateurs, soit 60 sénateurs. Lorsque la Rule XXII est appliquée, les sénateurs conservent toutefois le droit, pendant une heure, de présenter des amendements, des motions et demander la vérification du quorum, laissant au Président du Sénat le devoir de vérifier que ce temps de parole soit respecté (Rules $X X I I, 2,3^{e}$ alinéa). En absence d'un consentement unanime, aucun amendement n'est recevable. Toutefois, les Rules prévoient, dans certaines circonstances, la possibilité de présenter des amendements (amendements in first degree) ou des sous-amendements (amendements in second degree). En effet, à partir d'1h du jour suivant, il est possible de présenter des amendements, et $1 \mathrm{~h}$ avant le vote de la motion pour les sous-amendements. Ainsi, même en cas de vote d'une motion to close a debate, la clôture du débat peut se poursuivre aux calendes grecques. En effet, si tous les sénateurs non votants la motion (40 sénateurs) demandaient la parole pendant $1 \mathrm{~h}$, les débats se poursuivaient pendant presque deux jours (40 heures). Mais ces sénateurs pouvaient d'autre part présenter des amendements et des sous-amendements, retardant ainsi le vote du texte ${ }^{69}$. C'est pour cette raison qu'à partir de 1979, afin de pallier à ces inconvénients, les Rules ont mis en place une sorte de TLP pour débattre avant le vote final. II est prévu qu'après $36 \mathrm{~h}$ de débat l'on procède au vote après la vérification du quorum requis qui consiste en la majorité des sénateurs élus (Rules VI), faute de quoi aucun vote ne pourra avoir lieu (fin du $4^{\mathrm{e}}$ alinéa de la Rules VI). Le vote du texte $36 \mathrm{~h}$ après la motion to close the debate constitue un délai contraignant qui peut être prolongé d'une motion votée par les 3/5 des sénateurs lequel sera chargé de prévoir la date du vote final. Les Rules, sur ce point, se limitent à prévoir que si une telle motion est votée, le temps des débats sera reparti de manière proportionnelle entre la majorité et l'opposition.

À compter du 24 janvier 2013, a été apporté une modification à la Rule XXII qui prévoyait un cas de figure particulier pour une motion to close the debate. En effet, si une telle motion était signée par 30 sénateurs, correspondant à 16 sénateurs incluant le leader de la majorité et de la minorité, 7 sénateurs non rattachés à la majorité et 7 sénateurs non rattachés à la minorité, cette dernière était mise aux voix $1 \mathrm{~h}$ après le début de la séance successive (3 de la Rules $X X I I)^{70}$. En raison du nombre requis des sénateurs non rattachés à la majorité et minorité, un tel procédé n'a pu être mis en place ${ }^{71}$. Lors de la fin de la même année (21 novembre 2013) s'est mis en place un précédent permettant à une majorité

69 (https://www.rules.senate.gov/imo/media/doc/CDOC-113sdoc18.pdf).

70 Ibidem.

71 (http://www.senate.gov/senators/leadership.htm). 
(51 sénateurs) d'adopter une motion de clôture pour contrecarrer le filibustering par rapport aux nominations du président des États-Unis, à l'exception des juges pour la Cour Suprême, aux termes de l'article 2, section 2 de la Constitution américaine "le Président désignera et, sur l'avis et avec le consentement du Sénat, nommera les ambassadeurs, les autres ministres publics et les consuls, les juges de la Cour Suprême et tous les autres agents des États-Unis[...] ". En effet en raison de l'article 2 de la constitution américaine, il y a un concours collectif de deux acteurs : la désignation par le président des États-Unis, l'avis et le consentement du Sénat. Dans cette situation, le filibustering est un outil pour s'opposer et retarder le plus possible les nominations présidentielles. Néanmoins, ce dernier porte atteinte à l'exercice d'une prérogative constitutionnelle : celle du Sénat d'exercer son advice and consent et du Président de pouvoir utiliser son pouvoir de nomination ${ }^{72}$.

Le quorum requis pour l'approbation d'une motion constitue une garantie ; ainsi, le droit parlementaire existant au Sénat évite la tyrannie d'une simple majorité qui pourrait numériquement s'imposer à la minorité. Si le quorum ne s'apparente plus au quorum constitutionnel des $2 / 3$, il s'agit tout même d'un quorum de garantie. En effet, si l'on considère les sessions du Parlement américain qui ont une durée de 2 ans, le quorum pour faire recours à la motion était présent seulement dans 3 occasions. II s'agissait du $111^{\text {e }}$ Congrès $^{73}$ (2009-2011) où les démocrates disposaient de 61 sénateurs, même seuil atteint lors du 94e congrès (1975-1977) et $95^{\mathrm{e}}$ congrès (1977-1979) ${ }^{74}$. Si la révision de 1975 de la XXII Rules en abaissant la majorité qualifiée, était finalisée à rendre plus facile l'approbation de la cloture motion à 60 sénateurs, il est paradoxal d'observer comme le seuil précédent des $2 / 3$ fut davantage atteint dans la composition du Congrès que par rapport au seuil actuel. Le précédent quorum fut atteint 6 fois par les démocrates : le $74^{\mathrm{e}}$ congrès (1935-1937), 69 sièges ; le 75e Congrès (1937-1939), 76 sièges ;

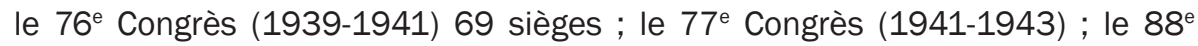
Congrès (1963-1965) ; 89e congrès (1965-1967), 68 sièges.

La centralité du bicamérisme américain est le résultat de la séparation stricte des pouvoirs qui se met en place par des délais temporaires différenciés. En effet, l'apparente homogénéité entre les scrutins électoraux des deux chambres, scrutin uninominal à un tour pour les représentants et les sénateurs, doit être mise en lien avec l'hétérogénéité existante pour l'attribution égalitaire des sièges sénatoriaux : 100 sénateurs sont élus dans 50 collèges électoraux

72 D. CONNIL, "La petite révolution américaine, quand le Sénat tente de limiter l'obstruction parlementaire ", Constitution, 2014, op. cit., p. 38.

73 II faut souligner que durant cette période les démocrates disposaient de 59 sièges. Le seuil de 60 sièges fut atteint grâce au sénateur Arlen Spencer. Ce dernier réélu en 2004 parmi les Républicains changea de camps politique en 2009, devenant un démocrate indépendant avant l'expiration de son mandat en 2010. C'est ainsi que ce seuil de 60 sièges eut une courte durée : à partir de 2010 les démocrates disposaient de 59 sièges puisque le sénateur Arlen ne fut pas réélu lors des ré-élection bild de 2010. 
(constituencies), correspondants aux 50 États fédérés, dans chaque collège sont élus deux sénateurs.

Cet aspect, en lien avec le renouvellement total de la Chambre des représentants tous les 2 ans et le renouvellement partiel du Sénat, rend possible la présence de majorités opposées entre les deux chambres du Congrès. En effet, par simplicité, en prenant en compte la période allant du $79^{e}$ Congrès (1945-1947), à 7 reprises on a eu des majorités différentes entre les deux chambres : le $97^{\mathrm{e}}$ congrès (1981$1983)$, le $98^{\mathrm{e}}$ congrès (1983-1985), le 99e congrès (1985-1987), le $112^{\mathrm{e}}$ congrès (2011-2013), le 113 (2013-2015) et l'actuel 116 eongrès (2019-2021).

La situation qui s'est produite lors du 107 e congrès (2001-2003) et du $110^{\mathrm{e}}$ congrès (2007-2009) est très particulière et permet de cerner le rôle important exercé par le Sénat dans le bicamérisme américain. En effet, pendant ces deux sessions, si à la chambre des représentants était présente à une majorité, au Sénat il y avait une situation d'égalité parfaite : 50 sénateurs pour les démocrates et les républicains lors du $107^{\mathrm{e}}$ congrès, 49 sièges pour les deux partis pendant le $110^{\mathrm{e}}$ congrès. Cette situation illustre bien le pouvoir important accordé au Vice-Président des États-Unis, le président du Sénat, car dans certaines circonstances son soutien s'avéra déterminant pour obtenir la majorité (il s'agit du tie breaking vote). Ce fut le cas pendant le $107^{\mathrm{e}}$ congrès qui a constitué une situation sans précédent et atypique au sein du Sénat. Lors de la courte période allant du 3 au 20 janvier 2001, le vote du Vice-Président sortant Al Gore permit aux démocrates d'atteindre les 50 sièges. Cet avantage fut de courte durée en raison du nouveau Vice-président républicain, Richard Cheney, qui fut déterminant pour la majorité républicaine (held the deciding vote) du 20 janvier au 24 mai. À partir de cette date, le sénateur républicain James Jeffords sortit du groupe républicain et devint un sénateur sans étiquette (independent senator) mais annonça son affiliation aux démocrates le 6 juin 2001, ce qui renversa les équilibres politiques, donnant une voix de plus aux démocrates. Toutefois, le 25 octobre 2002, le décès du sénateur Wellstone entraîna l'élection du sénateur républicain James Talent, modifiant à nouveau l'équilibre en faveur des républicains pendant le dernier mois de durée du congrès. La situation qui se produisit lors du $110^{\mathrm{e}}$ congrès fut un cas d'égalité apparente en raison de deux sénateurs indépendants, proches des démocrates, qui garantirent une majorité sénatoriale des démocrates de 51 contre 49 républicains.

Le tie breaking vote est important non seulement en cas d'absence de majorité au Sénat, mais aussi dans le cadre d'un vote décisif lorsqu'il y a une égalité dans des votations parlementaires. En effet, le républicain Cheney a exprimé 8 fois le tie breaking vote dans la période 2001-2009, le démocrate Al Gore 4 fois en 1993-2001. Selon les estimations faites par les bureaux du Sénat américain, dans I'histoire parlementaire américaine le tie breaking vote a été utilisé à 244 reprises. L'utilisation du tie breaking vote brise une pratique classique, 
politique ${ }^{75}$, du droit parlementaire qui exige que le président d'Assemblée n'exerce pas son droit de vote. Une telle pratique n'a pas de caractère obligatoire constant en France et fait l'objet d'une dérogation en présence de majorité très courte ; ce fut le cas le 7 décembre 1985 par M. Roger-Machart qui prit part au vote et le 6 mai 1986 par M. Mestre, mais aussi du président d’Accoyer lors de la révision de 2008.

Les exemples cités précédemment et le système d'élections des deux chambres avec ses hétérogénéités, nous témoignent la place centrale occupée par le Parlement américain et le concours déterminant du Sénat dans ses fonctions non législatives (adivce and control), ce qui permet à ce jour de renverser les propos prononcés par Woodroow Wilson se référant à la Chambre des représentants comme "le pouvoir dominant et même irrésistible du système fédéral "76.

\section{b. Le bicamérisme australien}

La structure de ce Parlement constitue un bel exemple de bicamérisme hybride qui a subi l'influence des modèles anglais et américain.

La House of representatives, composée de 150 députés, ayant pour finalité de représenter les électeurs des 6 États fédérés du pays en fonction de leur population : Australie méridionale, Australie occidentale, Nouvelle Galles du Sud, Queensland, Tasmanie, Victoria.

Le Senat est composé de 76 membres élus à la hauteur de 12 sénateurs pour chaque État.

Comme aux États-Unis, le mandat des deux chambres est différent : 3 ans pour la chambre des représentants et 6 ans pour les Sénat qui se renouvelle de la moitié des sièges tous les 3 ans. L'hétérogénéité ne vise pas seulement la durée des chambres, mais aussi le système électoral : scrutin majoritaire uninominal pour la House of Representatives, scrutin proportionnel à partir de 1949 pour le Senat.

Le rôle et les fonctions de la deuxième chambre la rapproche plus du modèle américain que de celui de Westminster. En effet, après les deux Parliaments Acts, la House of Lords ne put exercer son dernier mot lors de la procédure législative. Les prérogatives du sénat australien sont accrues en raison du scrutin proportionnel. Ce dernier favorise la représentation des petits partis ou indépendants. C'est ainsi qu'en raison du scrutin électoral différent, la majorité présente au sénat est hétérogène par rapport à celle existante à la première chambre, généralement le parti majoritaire ne dispose pas de la majorité absolue dans cette assemblée. Dans ces circonstances sont déterminants les crossbenchers, des partis indépendants appelés ainsi car ils siègent au milieu de

75 C'est dans ces termes qu'est classée la pratique qui prévoit que le Président ne participe pas aux scrutins en raison de son obligation de neutralité. Cf., P. AVRIL, J. JICQUEL, J.-E. GICQUEL, Droit parlementaire, LGDJ lextenso editions, $5^{\mathrm{e}}$ éd., op. cit., p. 19. 
I'hémicycle entre la majorité et l'opposition. Sous la législature courante, ils sont 14 : Australian Greens (9), Centre Alliance (2), Pauline Hanson's One Nation (2), Jackie Lambie Network $(1)^{77}$.

En plus des différences précédemment mentionnées, les deux chambres ont aussi des règles divergentes quant à la dissolution anticipée. Selon l'article 5 de la constitution australienne, la House of Representatives peut être dissoute, sur proposition du Premier Ministre, par le Gouverneur Général, qui aux termes des articles 2 et 61 de la Constitution exerce de iure le pouvoir exécutif au nom de la Reine Élisabeth II mais de facto ce dernier est exercé par le gouvernement en place qui d'ailleurs, désigne le Gouverneur, formellement nommé par la Reine. S'agissant de la dissolution de la première chambre, il y a ainsi une simple dissolution, qui répond aux règles classiques de la dissolution à l'initiative gouvernementale. À noter qu'aucune délibération de la House of Representative n'est nécessaire, la procédure s'apparente à celle existant au Royaume-Uni avant le Fixed Parliamentary Act de 2011.

Pour la dissolution du Senat, la constitution prévoit une procédure particulière (double dissolution) régie par l'article 57 qui établit un lien entre procédure législative et dissolution parlementaire.

En effet, tout texte approuvé par la Chambre des représentants est envoyé au Sénat. Si celui-ci rejette, n'approuve pas, ou approuve avec des modifications le texte, ce dernier est renvoyé à la Chambre des représentants.

Si cette dernière ne valide toujours pas encore le texte, la procédure est suspendue pendant 3 mois.

À l'issue de cette période de réflexion finalisée à asseoir un compromis, l'examen du texte repart à la Chambre des représentants. Si celle-ci confirme son texte, ce dernier est envoyé au Sénat pour un deuxième examen. En cas d'amendement ou de rejet par le Sénat, le texte est renvoyé à la Chambre des représentants.

Si le désaccord persiste toujours le Gouverneur Général peut dissoudre les deux chambres et organiser des élections anticipées. Suite aux élections, si la House of Representatives réitère et approuve à nouveau le texte voté avant la dissolution, ce dernier est transmis au Sénat. En cas de lecture infructueuse, le texte est à nouveau retransmis à la House pour une dernière lecture.

Si l'échec persiste encore, on procède à la convocation du Parlement en séance commune (Joint Sitting). Ainsi, le dernier texte approuvé par la House fera l'objet d'un vote à la majorité absolue des membres. En cas d'approbation, le texte sera considéré comme définitivement approuvé.

On constate que la procédure législative australienne ne prévoit pas formellement d'organe pour asseoir un compromis, à l'instar d'autres pays ayant un bicamérisme égalitaire (Suisse, Bosnie-Herzégovine, États-Unis).

Malgré le silence de l'article 57, au début furent mises en place des

77 Cf., (https://www.aph.gov.au/About_Parliament/Senate). 
commissions de conciliation (conferences). Dans l'histoire australienne, 3 commissions se réunirent avec succès en 1921-1922, en 1930 et 1931. Comme la convocation de ces commissions n'empêchait pas le Sénat de modifier le texte, ce dernier décida de s'appuyer sur l'expérience du Parlement de l'Australie du Sud où avait été remarquée l'inutilité de ces conférences, car ses représentants, lors des réunions, reportaient toujours la décision finale ${ }^{78}$. C'est ainsi que cet organisme tomba en désuétude et ne se réunit plus en raison du contrôle que le gouvernement exerçait sur la House of Representatives. Face à cette situation, il parut plus efficace pour les sénateurs de négocier avec ce dernier la législation en cours.

II faut néanmoins préciser que la progressive désuétude à utiliser les conférences n'eut aucun effet sur le droit codifié, car de iure le mécanisme des conférences est toujours présent dans les Standing Orders du Senate aux articles 156-162, mais il demeure de facto inutilisé alors qu'il pourrait être toujours envisagé pour rapprocher les points de vue entre les deux chambres. La demande doit venir de la deuxième chambre australienne par un message transmis aux représentants dans lequel sont indiqués les 5 sénateurs qui vont composer cet organisme (les managers) avec 5 autres représentants ${ }^{79}$. On peut imaginer qu'à l'issue de ces travaux puisse avoir lieu un vote en raison du verbe employé (to confer, s'accorder), même si la rédaction de l'article 160 des standing orders alinéa 2 discipline les travaux de manière très générique:

"Lors des conférences les membres du Sénat doivent indiquer les motifs ou les modifications du Sénat aux membres de la chambre des représentants, ou recevoir par ces derniers les motifs ou les modifications de la Chambre des représentants, après quoi les membres du Sénat présents dans cette conférence pourront s'accorder librement avec les représentants " 80 .

En raison de la non application de ces règles parlementaires, la procédure législative consiste en une tentative de compromis entre les deux chambres.

Le mécanisme de la dissolution est à éviter un blocage parlementaire permanent (permanent deadlock between the Houses). Néanmoins, il repose sur un pari de la majorité présente à la House of Representatives d'être à nouveau majoritaire dans cette chambre afin de pouvoir avoir recours in fine au Joint Sitting du Parlement. C'est ainsi que la dissolution devient un outil de rationalisation de la procédure législative pour mettre fin à un différend dont l'objet repose sur

78 Cf.,(https://www.aph.gov.au/About_Parliament/Senate/Powers_practice_n_procedures/Odgers _Australian_Senate_Practice/Chapter_21).

79 (https://www.aph.gov.au/Parliamentary_Business/Chamber_documents/Senate_chamber_documents/standingorders).

80 Article 160 Standing Orders paragraph (2) "At conferences the managers for the Senate shall deliver the reasons or resolutions of the Senate to the managers for the House of Representatives, or receive from the managers for the House of Representatives the reasons or resolutions communicated by the latter, after which the managers for the Senate shall be at liberty to confer freely with the managers for the House of Representatives ". Cf., (https://www.aph.gov.au/Parliamentary_Business/Chamber_documents/Senate_chamber_documents/standingorders). 
une législation hostile qui ne recueille pas le consentement des sénateurs. Cette dissolution ne donne aucune garantie. En effet, si la majorité est battue à la House of Representatives, la législation sera abandonnée. La dissolution s'apparente à un référendum sur le gouvernement car les élections qui seront organisées vont tourner autour de la législation contestée : l'exécutif va inciter ses électeurs à voter en sa faveur pour permettre l'approbation de la dite législation. De leur côté, les oppositions sénatoriales pourront externaliser leur opposition parlementaire face à l'électorat qui aura le dernier mot : un vote pour la coalition demandant la double dissolution consistera en un vote pour la politique menée et la législation contestée par les sénateurs ; en revanche, un vote pour les oppositions consistera dans une censure électorale sur le gouvernement demandant la dissolution. Si de iure la dissolution entraîne l'organisation de nouvelles élections, dans cette circonstance il s'agit d'élections anticipées dont la question soumise aux électeurs est de savoir s'ils approuvent ou non la législation contestée.

Le mécanisme retenu à l'article 57 de la constitution est une concession $d u$ fédéralisme à la démocratie car, en raison de profonds désaccords parlementaires, les représentants vont prévaloir sur la représentation géographique exprimée par le sénat.

Si on observe de manière attentive la procédure législative telle qu'elle ressort de l'article 57, on peut affirmer que le bicamérisme australien n'est pas totalement égalitaire. II s'agirait en effet d'un bicamérisme égalitaire tempéré car , les deadlocks entre les deux chambres arrivent à leur terme par le Joint Sitting qui constitue une sorte de dernier mot de la House of Representatives en raison de la primauté numérique des représentants. Dans cette circonstance, le bicamérisme s'avère inégalitaire. En revanche, en cas de défaite de la coalition demandant la double dissolution, le bicamérisme ne peut être considéré comme étant égalitaire car les électeurs rejettent les motifs de la dissolution et expriment un avis défavorable au texte contesté par les sénateurs mais soutenu par l'exécutif.

Par ailleurs, une analyse de l'article 57 fait percevoir que la procédure législative dessinée permet in fine aux représentants de s'imposer face aux sénateurs.

Une telle affirmation doit être nuancée par la pratique qui a été faite des doubles dissolutions. Sur les 7 dissolutions qui ont eu lieu (1914, 1951, 1974, 1975, 1983, 1987, 2016) seule celle de 1974 a permis au Joint Sitting d'approuver définitivement le texte voté par les représentants et, contesté par les sénateurs. Celle de 1951 où le Gouvernement libéral Menzies (1949-1966), au lieu de reproposer le texte sur lequel avait été demandé la double dissolution, approuva d'autres mesures d'ordre similaire, soulevant ainsi de doutes alors sur la dissolution organisée. En revanche, les dissolutions de 1914, de 1975 et 1983 furent inefficaces en raison de la défaite du gouvernement qui avait demandé la double dissolution. La dissolution de 2016 ramena au pouvoir le gouvernement Turnbull (2015-2018) qui disposait alors d'une majorité d'un seul représentant et n'avait aucune majorité au Sénat. Ce fut grâce à des compromis avec les 
20 crossbenchers, le nombre le plus élevé de l'histoire australienne, que le gouvernement approuva la loi à l'origine de la dissolution.

Compte tenu de sa position forte, le Sénat australien constitue un check and balance à une Chambre basse conçue sur le modèle Westminster. Ce qui explique que les tentatives de révision constitutionnelle finalisée à réduire les prérogatives du Sénat ${ }^{81} \mathrm{ou}$ de modifications législatives visant à aligner le scrutin électoral des sénateurs à celui des représentants. Si le Sénat constitue un check and balances constitutionnel mis en place par la procédure législative, un deuxième sera été mis en place par l'adoption d'une législation électorale proportionnelle. La Constitution de son côté (article 7, alinéa 2) ne fixe pas un nombre déterminé de sénateurs par État, mais fixe les critères directifs pour les éventuelles lois adoptées par le Parlement qui pourra augmenter ou diminuer le nombre de sénateurs, tout en respectant le principe que " les États aient tous une représentation numériquement égale [...] ". En raison de ce principe qu'une éventuelle modification de la législation électorale en faveur d'un scrutin majoritaire à l'instar de celui en place à la House of Representatives ne pourrait in toto empêcher la formation de majorités opposées entre les deux chambres. En effet, ce n'est pas seulement le différent scrutin électoral qui permet la formation de majorités opposées, mais aussi le principe de l'élection égalitaire des sénateurs pour tous les États en lien avec la durée différente des mandats de représentants et sénateurs, à l'instar du modèle américain : les scrutins électoraux pour les deux chambres demeurent identiques mais diffèrent quant à la représentation égale des sénateurs pour tous les États fédérés. II faut aussi ajouter qu'une éventuelle introduction d'une loi électorale majoritaire pour le Senat risquerait de transformer ce dernier en rubber stump ce qui implique, dans la tradition parlementaire australienne, une chambre se limitant à voter en faveur d'un texte sans l'examiner en profondeur, altérant ainsi sa fonction de check and balances.

Si la double dissolution est l'instrument principal permettant à l'exécutif de mettre fin à un deadblock parlementaire en cas d'opposition sénatoriale, il pourrait aussi utiliser la simple dissolution pour la House of Representatives Iorsqu'il n'a pas de majorité dans cette assemblée mais une majorité stable au sénat. En effet, il pourrait arriver que suite à de nouvelles élections, seul le Senat exprime une majorité. Dans cette circonstance, l'article 57 de la Constitution serait inapplicable car, en l'absence de majorité chez les représentants, à moins de s'appuyer sur des crossbenchers, il serait impossible pour l'exécutif de demander une double dissolution. Ainsi, la simple dissolution reposerait sur l'espoir de l'exécutif d'obtenir une majorité, même courte, à la House of representatives. Inversement, il serait probable que la simple dissolution donne lieu à une double dissolution. En effet, si une coalition politique disposait de la majorité au Sénat

81 Cinq référendums ont eu lieu entre 1967 et 1988 mais ils ont tous été rejetés par les corps électoral. 
mais pas à la Chambre des représentants, et que la seule dissolution de cette dernière n'aligne pas les deux majorités parlementaires, on se trouverait dans la situation d'avoir la House of representatives nouvellement élue exprimant une majorité opposée à celle du Sénat. Dans ce cas, la double dissolution serait probable, à moins que l'exécutif ne puisse s'appuyer sur un nombre suffisant de représentants crossbenchers.

La procédure législative australienne et la double dissolution reposent sur le pari du gouvernement d'être encore majoritaire à la House of representatives. Le mécanisme de rationalisation imaginé à l'article 57 (Joint Sitting) repose sur l'espoir de l'exécutif d'obtenir le soutien des électeurs pour approuver un texte hostile aux sénateurs. Néanmoins, le corps électoral se prononce lors d'élections anticipées et pas sur le deadblock subsistant entre les deux chambres. Le mécanisme retenu à l'article 57, en raison de la consultation des électeurs, s'apparente à celui imaginé en Italie lors des travaux de l'Assemblée constituante qui accoucha d'un bicamérisme égalitaire : afin de mettre fin au régime des navettes, les constituants avaient imaginé un mécanisme de consultation électorale.

En effet l'article 70 du projet de constitution de la République italienne prévoyait que :

"Les textes approuvés par une Chambre sont transmis à l'autre, qui doit se prononcer dans un délai de 3 mois. Lorsqu'une Chambre ne se prononce pas dans le délai prévu ci-dessus sur un texte approuvé par l'autre, ou lorsqu'elle rejette le texte, le Président de la République peut demander à cette chambre qu'elle se prononce à nouveau sur ce texte ou qu'elle le réexamine. Si elle ne prononce pas ou si avec la nouvelle délibération demandée elle confirme sa précédente, le Président de la République peut organiser un référendum sur le texte non approuvé "82.

Un tel mécanisme devait être la solution pour rationaliser le bicamérisme égalitaire italien.

Dans ce procédé, on constate un échafaudage constitutionnel d'étapes ayant pour finalité de parvenir à une solution. C'est notamment laxisme d'une chambre à ne pas se prononcer ou son rejet qui enclenche la procédure. Si une de ces deux circonstances se produit, la $2^{\mathrm{e}}$ étape consiste en une demande venant du président de la République adressée à la chambre responsable du deadblock. Cette étape est toutefois éventuelle car elle repose sur un pouvoir discrétionnaire du président de la République qui peut demander un nouvel examen à la chambre en question. En effet, l'absence de demande du président de la République entraînerait un enlisement de la procédure. Lorsqu'intervient cette $2^{\mathrm{e}}$ étape, s'ouvre une $3^{\mathrm{e}}$ étape qui consiste dans un réexamen adressé à la chambre responsable du blocage. Cette étape peut être la dernière dans l'improbable circonstance où la chambre décide de revenir sur la décision prise préalablement. Si la chambre maintient sa précédente délibération (un rejet du texte ou une approbation avec modification),

82 (http://legislature.camera.it/_dati/costituente/lavori/ddl/00nc.pdf). 
s'ouvre une éventuelle $4^{\text {e }}$ étape car l'organisation du référendum est une possibilité qui peut être exercée par le président de la République. Cette dernière étape consiste en la tenue d'un référendum sur le texte à l'origine du deadblock où les électeurs devront se prononcer par un oui ou un non.

À l'instar de la solution australienne, la solution du deadblock en Italie serait passée par une consultation des électeurs. On constate toutefois que la solution envisagée initialement prévoyait un rôle arbitraire du président de la République qui pouvait demander un réexamen ou une $2^{\mathrm{e}}$ délibération, ou organiser un référendum. L'absence de conditions dans lesquelles se serait exercé ce pouvoir aurait entraîné un pouvoir discrétionnaire excessif du Président qui, conformément à la constitution, doit être neutre et impartial en raison de son élection au suffrage indirect (article 79 du projet de la constitution, actuel article 83). De plus, il faut ajouter que l'organisation d'un référendum sur un texte sur lequel les chambres n'arrivent pas à s'accorder, risque de mettre en place un conflit entre le Parlement et la volonté populaire. En effet le Parlement doit pouvoir exercer librement sa fonction législative sans que les électeurs tranchent sur les éventuels désaccords parlementaires. Pour ces raisons, le mécanisme envisagé par les constituants italiens n'a pas été retenu.

Si la consultation des électeurs (double dissolution, référendum) sur le texte faisant l'objet d'un deadblock peut avoir un sens dans un bicamérisme, où les deux chambres se renouvellent à échéances hétérogènes, rendant plus probable la formation de majorités hétérogènes, en présence de mandats homogènes il est opportun de prévoir des limites à un éventuel référendum, à l'instar du modèle australien, et une procédure parlementaire permettant un délai de réflexion entre les deux chambres pour asseoir un compromis. C'est sur la base de ce principe que l'article 57 de la constitution australienne prévoit un délai de suspension de 3 mois pour permettre une réflexion supplémentaire.

La complexe procédure retenue en Australie prévoit un contrôle de la part de la Cour Suprême qui, par sa jurisprudence (Victoria vs Commonwealth, 1975 7 ALR 2), a précisé que l'intervalle de 3 mois est mesuré à compter du rejet du Sénat ou de son approbation avec modifications et non pas à partir de la $1^{\text {re }}$ lecture à la House of Represenatives ${ }^{83}$. Le non-respect de ce délai, vérifié par la Cour Suprême, frappe d'inconstitutionnalité la loi approuvée. En 1974 notamment, la haute juridiction australienne émit une censure contre une loi, créant une autorité pour le pétrole et les minéraux, loi approuvée par le gouvernement Whitlam après une double dissolution. Le grief d'inconstitutionnalité concernait le non-respect du délai constitutionnel des 3 mois incluant le $1^{\text {er }}$ rejet ou avis contraire du Senat et le $2^{\mathrm{e}}$ rejet ou avis contraire de la House of Representatives. En effet, lorsque surgit un deadblock, ce dernier requiert au moins deux rejets

83 Cf., Chapiter 21, Relations with the House of represenatives and the Senate, Australian Parliament. 
sénatoriaux pour pouvoir procéder à la double dissolution ${ }^{84}$, même si, à ce jour, cette interprétation de l'article 57 n'a pas encore été validée par la jurisprudence de la Cour Suprême.

En raison de cette procédure extrêmement sophistiquée, on constate que le nombre potentiellement illimité de doubles dissolutions peut s'appliquer à n'importe quelle loi. Néanmoins, comme l'a affirmé une part de la doctrine australienne, le pouvoir de dissolution doit être conçu comme un pouvoir de réserve de la constitution utilisé lorsque entrent en jeu d' importantes questions de politique législative ${ }^{85}$. Néanmoins, dans cette circonstance le rôle joué par la constitution est important en faisant appel au sens de responsabilité du pouvoir politique à ne pas abuser de certains instruments. En effet, lorsqu'une constitution reste muette sur l'utilisation de certains instruments, il n'est pas étonnant que le politique profite de certaines failles constitutionnelles, comme en témoigne le contexte français avant la révision de 2008 mettant en place une utilisation restrictive de l'article 49. 3.

La double dissolution donne ainsi de facto un pouvoir de domination de l'exécutif envers le Senat et la House of Representatives malgré les différentes issues des sept dissolutions qui ont eu lieu dans la pratique.

On constate donc un mécanisme de rationalisation dans cette procédure législative qui ne fait plus recours à l'équivalent américain des Conference Committee, où l'exécutif dispose de deux armes utilisables : la double et la simple dissolution.

\section{B. La double confiance du Parlement Italien}

La nécessité pour l'exécutif de bénéficier de la confiance des deux assemblées composant le Parlement, l'avis conforme est nécessaire, reproposent aujourd'hui, avec une grande actualité, les critiques soulevées par Sieyès lors des travaux de la constituante française de 1791 : s'il n'y a qu'une Nation et un seul peuple souverain, il est impossible de diviser en deux la volonté générale ; et même en admettant qu'une telle division soit possible, la deuxième chambre ne pourrait être qu'un doublon de la première. C'est ce qui rend inévitable la mise en place de mécanismes pour trouver une solution aux conflits entre les deux chambres. Chose qui se produit d'ailleurs dans la majorité des bicamérismes égalitaires contemporains où l'on assiste à la mise en place d'une rationalisation des procédures, réunion des membres des deux chambres pour asseoir un compromis (Suisse, États-Unis, Bosnie-Herzégovine), mais qui n'est pas une règle absolue car certains pays prévoient d'autres mécanismes pour solutionner les conflits (Australie, double dissolution) et d'autres n'en

84 Cf., not. cit., point 12 du Chapiter 21.

85 J. QUICK, The Legislative Powers of the Commonwealth and the States of Australia, 1919, p. 641. 
prévoient aucun (Italie).

À ce jour la généralité des bicamérismes contemporains n'a pas répondu au dilemme proposé par Sieyès et les ordres juridiques se sont limités à mettre en place un ensemble de règles : différenciation de la composition et de la durée de la deuxième chambre par rapport à la première ; exclusion de la deuxième chambre du rapport de confiance dans les pays adoptant une forme de gouvernement parlementaire classique ou une forme hybride, mélangeant éléments présidentiels et parlementaires ; limitation des pouvoirs de la deuxième chambre.

Sur tous ces aspects, on constate que le bicamérisme italien constitue un cas tout à fait singulier en raison des différences existantes avec les pays qui adoptent un bicamérisme inégalitaire, mais surtout avec ceux qui ont un bicamérisme égalitaire, notamment pour l'exercice paritaire de la fonction de contrôle et de confiance envers l'exécutif.

En effet, si dans la doctrine italienne d'après guerre, en référence au bicamérisme s'est mise en place la distinction entre bicamérisme égalitaire (ou parfait) et bicamérisme d'atténuation (ou imparfait) ${ }^{86}$, il faut observer que la qualification du bicamérisme étant égalitaire ne peut dériver de l'articulation interne du système bicaméral, que par les compétences attribuées aux deux chambres au niveau de la fonction législative, du rapport de confiance entre le législatif et l'exécutif, etc. C'est ainsi qu'il n'existe pas in toto un bicamérisme égalitaire, mais un bicamérisme égalitaire par rapport à certaines fonctions : il en va ainsi du bicamérisme américain, australien, suisse, et de la BosnieHerzégovine. Dans d'autres fonctions, ce dernier ne demeurera pas inégalitaire.

À ce sujet, il est important d'effectuer une analyse des fonctions exercées par les deux chambres, qu'elles soient égalitaires ou inégalitaires. De cette manière, il convient davantage de faire une distinction entre bicamérisme égalitaire absolu et bicamérisme égalitaire relatif. En effet, les bicamérismes égalitaires contemporains sont relatifs car c'est principalement la fonction législative qui est exercée par les deux chambres sur un pied d'égalité, de même la fonction de révision constitutionnelle. En référence à cette dernière, on constate que la quasi-majorité des bicamérismes sont égalitaires ${ }^{87}$, les chambres y concourent de manière paritaire ${ }^{88}$. II s'agit en effet d'une règle générale qui prévoit quelques

86 Cf., E. SPAGNA MUSSO, Bicameralismo e riforma del Parlamento, dans Parlamenti, istituzioni, democrazia, Giuffrè, Milano, 1980, p. 124.

87 Les pays où la deuxième chambre peut utiliser son pouvoir de blocage sont nombreux même en dehors du continent européen, c'est le cas du Chili (art. 127 Constitution), du Gabon (art. 116 Constitution), du Maroc (art. 172 Constitution) et de l'Algérie (art. 174).

88 Sur ce point un veto absolu peut être exercé par la House of Lords pour rejeter les textes de lois visant à proroger la durée de la législature parlementaire au-delà des termes ordinaires - 5 ans - ou de réduire la durée maximale du mandat parlementaire : ainsi se prononce P. LEYLAND, Introduzione, op. cit., pp. 79-80 ; M. RUSSEL, The British House of Lords, op. cit., p. 79-80. Sur ce point on serait en présence d'un genre de loi à compétence égalitaire où la Chambre Haute anglaise peut empêcher la Chambre basse d'utiliser son dernier mot. En fonction de cette hypothèse, on pourrait envisager être en présence de loi à valeur constitutionnelle, même si cette terminologie dans le contexte anglais n'a pas de sens car il n'existe pas de lois 
exceptions. L'une d'entre elles est représentée par le cas australien. En effet, la révision constitutionnelle australienne, aux termes de l'article 128, prévoit deux procédures, l'une paritaire et l'autre inégalitaire : l'approbation par les deux chambres à la majorité absolue et la tenue d'un référendum pour approbation avec le critère de la double majority, l'approbation par une seule chambre d'un texte après deux délibérations dans un délai de 3 mois à compter d'un rejet ou de la non approbation du texte par l'autre chambre ${ }^{89}$.

À propos des pays ayant un bicamérisme égalitaire (v. supra A du II), les deux chambres partagent ax aequo la fonction législative.

À l'exception des États-Unis et de la Suisse où, en raison de la forme de gouvernement existante, le législatif ne peut exercer une forme de contrôle contraignant à l'égard de l'exécutif au moyen d'instruments comparables à la motion de censure ou de défiance, dans les autres pays où subsiste une forme de gouvernement parlementaire, la deuxième chambre est placée dans une position d'inégalité par rapport à la première.

Une exception sur ce point pourrait être constituée par les Pays-Bas en raison de l'attribution aux deux chambres du pouvoir de contrôle à l'égard de l'exécutif, qui n'est pas ouvertement discipliné par la Constitution mais de nature conventionnelle (v. supra). Ces conventions annulent en pratique une éventuelle égalité des deux chambres face au contrôle de l'exécutif. Si la pratique parlementaire a rendu possible la mise en place d'instruments pouvant induire le gouvernement à la démission (v. supra p. 57) dans les deux chambres ${ }^{90}$, en raison de l'élection au suffrage indirect des membres de la deuxième chambre, celle-ci exercera avec beaucoup de prudence ses prérogatives, notamment le contrôle envers l'exécutif et le rapport de confiance, qui se met en place de facto entre le gouvernement et la chambre élue au suffrage direct ${ }^{91}$.

ordinaires ou constitutionnelles. II faut en plus préciser que cette définition (loi constitutionnelle) dépend de l'ordre juridique auquel on se réfère. En Italie, toutes les lois prévoient un concours paritaire des deux chambres dans l'élaboration du texte mais ce n'est pas pour cette raison qu'on parle de lois constitutionnelles, lesquelles sont régies par une procédure renforcée (art. 138) par rapport à celle des lois ordinaires (art. 70). En France, il y a des lois où les deux chambres exercent sur le même pied d'égalité leurs compétences (art. 46, avant dernier alinéa et la loi organique visée à l'article 88-3 C) sans être des lois constitutionnelles. En effet, le concept de loi constitutionnelle dépend autant de la dimension matérielle de la loi que de sa dimension formelle qui prévoit une procédure plus complexe par rapport à celle des lois ordinaires.

89 Une autre exception est constituée par la Slovénie où aux termes des articles 168-169 de la constitution "la révision constitutionnelle est décidée seulement par l'Assemblée Nationale, à la majorité des 2/3 ". Cf., C. DECARO, I bicameralismi, op. cit., p. 27. Un cas, sui generis, est constitué pr le Lesotho, art. 85 de la Constitution qui prévoit non pas une procédure mais des quorums différents pour l'approbation des lois constitutionnelles : la majorité absolue des membres de la chambre basse, la majorité simple des membres du Sénat.

90 R.-B ANDEWEG, G.-A. IRWIN, Governance and Politics, op. cit., p. 173 qui souligne " it is generally accepted that a motion of no confidence, if adopted, cannot be ignored and must result in the resignation of the minister or of the Cabinet as a whole, or in the dissolution of Parliament ".

91 Le rôle secondaire de la deuxième chambre est témoigné aussi par la pratique de l'accountability day. Le troisième mardi de mai de chaque année, le Ministre de l'Économie présente un rapport économique de la dernière année financière, permettant ainsi au Parlement de se prononcer sur 
Le seul pays qui prévoit des prérogatives exercées de manière paritaire entre les deux chambres est la Bosnie-Herzégovine. En effet, la forme de gouvernement parlementaire de ce pays prévoit la nomination du président du Conseil des Ministres qui, pour former son exécutif, doit obtenir la confiance de la Chambre des représentants. Cette confiance s'avère nécessaire aussi pour la nomination des Ministres (article IV, 4, $1^{\text {er }}$ alinéa dernière phrase et b.). Si la confiance pour la formation du gouvernement est nécessaire seulement où la Chambre des représentants, le gouvernement doit bénéficier de la confiance des deux chambres qui peuvent à tout moment approuver une motion de censure (article IV, 4, c.) C'est ainsi que le mécanisme de confiance retenu dans la constitution de la Bosnie-Herzégovine s'avère atypique car il prévoit, dans un premier temps, un mécanisme de rationalisation (confiance initiale) qui se met en place par le vote de la seule Chambre des représentants. Ensuite l'exécutif doit bénéficier de la confiance des deux chambres (double confiance) qui peuvent à tout moment la lui révoquer. La Chambre des peuples peut déposer une motion de censure signée par les 3 délégations (bosniaque, serbe, croate) aux termes des articles 141-142 de son règlement qui devra faire l'objet d'un vote dans les 30 jours successifs à son dépôt ${ }^{92}$. Un mécanisme similaire est prévu par le règlement de la Chambre des représentants (articles 143-147).

C'est pourquoi la procédure qui discipline la confiance de l'exécutif envers le législatif dans la constitution bosniaque ne peut s'apparenter à celle italienne. En effet, dans le régime parlementaire italien l'exercice paritaire des deux chambres dans la confiance place ces dernières immédiatement sur un pied d'égalité parfaite. La constitution est claire sur ce point, aucun obiter dictum ne peut être invoqué, comme le prévoit l'article 94 alinéa 1 "Le Gouvernement doit obtenir la confiance des deux chambres " et l'alinéa 2 "Dans les dix jours suivant sa formation, le gouvernement se présente devant les chambres pour obtenir leur confiance ", contrairement à ce que stipule l'article IV, 4 premier alinéa 1 de la constitution bosniaque "Le président du Conseil des Ministres prend ses fonctions avec l'assentiment de la Chambre des représentants "93.

Par l'analyse comparative des régimes bicaméraux des États Européens, on constate que l'attribution de compétences identiques dans le rapport de confiance est une singularité propre au système bicaméral italien. C'est sur cet aspect, avec les autres fonctions qui sont exercées aussi de manière égalitaire, que l'on peut affirmer la singularité de ce bicamérisme qui en fait un cas unique en son genre. L'inclusion du Sénat italien dans le rapport de confiance est liée à l'élection directe de ses membres et à sa représentation politique. Une analyse comparative ne permet pas d'élever cette affirmation à une règle générale car de

la mise en place du programme gouvernemental. Il est intéressant d'observer que ce débat se déroule face à la chambre Tweed Kamer. Cf. La Costituzione in movimento, op. cit., 77.

92 Cf., (http://www.parlament.ba/Content/Read/37?title=Poslovnik\&lang=bs).

93 Cf., Constitution Finder, Constitute- The World's constitutions to read, search, and compare, Professor Jones, University of Richmond. 
nombreux contextes constitutionnels européens qui mettent en crise le principe pour lequel l'élection directe de la deuxième chambre est toujours une condition sine qua non pour que cette dernière soit incluse dans le (double) rapport de confiance : ce qui a été observé (v. supra) pour le cas polonais et de la République Tchèque. Si on constate l'ensemble des pays ayant une forme de gouvernement parlementaire et semi-présidentiel, il est possible d'affirmer la présence de bicamérismes égalitaires relatifs car le rapport de confiance demeure une prérogative réservée à la chambre élue au suffrage universel direct, c'est-à-dire celle qui assure la représentation politique générale.

Le bicamérisme italien assume une dimension politique car la représentation de la deuxième chambre est cohérente avec le principe démocratique. En effet , ce type de bicamérisme montre la tendance à intégrer la volonté populaire représentée dans la première chambre. Si le critère politique est à la base de nombreuses constitutions (Italie, Chili, République Tchèque, Roumanie, Japon, Thaïlande $\left.{ }^{94}\right)$, ce dernier n'implique pas l'attribution de compétences identiques à la deuxième chambre du Parlement. Toutefois, on constate que certaines instances propres au pluralisme sont sacrifiées pour parvenir à un compromis entre forces politiques. Ce qui, in fine, permet de dire que ce type de compromis " rend impossible de donner un fondement rationnel au bicamérisme italien "95. Le compromis politique atteint en Assemblée constituante accoucha d'une deuxième chambre qui ne correspondait à aucun modèle précédent ${ }^{96}$.

Le bicamérisme introduit devait correspondre à une logique de checks and balances qui se mettaient en place non pas par une composition différente, en raison de faibles dispositions constitutionnelles de différenciation, mais par deux éléments : la première composition transitoire lors de la lre législature républicaine (III Disposition Transitoire et Finale de la Constitution italienne) qui mit en place uniquement de 1948-1953 un Sénat avec une composition différente de la Chambre des députés (v. supra); la durée initialement différente des deux chambres.

La durée différente du Sénat était le réel motif devant justifier la double confiance prévue dans le bicamérisme égalitaire absolu italien.

Une première motivation était d'ordre conservateur. Le Sénat de la République devait constituer un prolongement idéal du défunt Sénat du Royaume du Statut Albertin (v. supra pages 12-13). En effet, le Sénat était une assemblée

94 Cf., article 57 constitution italienne ; art. 49 de la Constitution chilienne ; art. 18, alinéa 2 de la Constitution tchèque ; art. 62, alinéa 1 de la constitution Roumaine ; art. 46 de la Constitution japonaise ; art. 111 de la Constitution thaïlandaise.

95 Cf., G. RIVOSECCHI, La lezione dell'Assemblea Costituente sui processi di rappresentanza : verso un integrazione della rappresentanza politica ?, in C.DECARO (a cura di), II bicameralismo in discussione : Regno Unito, Francia Italia.Profili comparati, LUISS University Press, Roma, 2008, op. cit., p. 171. Du même avis, G. FERRARA, Art. 55, in G. BRANCA (a cura di), Commentario della Costituzione. Le Camere. Art. 55-63, tomo 1, Zanichelli-Società Editrice del Foro Italiano, Bologna-Roma, 1984, op. cit., p. 1.

96 Cf., S. MATTARELLA, Il bicameralismo, op. cit., pages 1162-1163. 
permanente, contrairement à la Chambre des députés ; c'est ainsi que cette différence temporaire était une manière de mettre en place une supériorité temporaire accrue par rapport à la Chambre des députés. C'était ainsi un correctif aristocratique comme celui qui prévoyait la présence de sénateurs de droit et à vie et les sénateurs nommés par le Président de la République (Sénateurs Viagers, article 59 alinéa 1 et 2 de la constitution).

Mais c'est surtout le deuxième motif d'ordre social qui devait légitimer d'avantage le Sénat à donner la confiance au gouvernement. En effet, la durée différente du Sénat aurait permis de le lier davantage aux évolutions sociales et aux équilibres politiques du pays. Sa durée différente aurait permis soit de confirmer ces équilibres politiques ou soit de les altérer dans une fonction rééquilibrante, évitant ainsi la tyrannie de la première chambre.

La loi constitutionnelle $n^{\circ} 2 / 1963$, en alignant la durée des deux chambres, élimina leurs faibles différences. En effet, la double confiance existante risque d'être un instrument inutile, voir répétitif et déstabilisant pour l'action du gouvernement.

La fonction modératrice du Sénat devient ainsi imperceptible, voire invisible, du fait qu'il exerce in toto les mêmes compétences que la Chambre des députés.

Le bicamérisme égalitaire absolu existant en Italie souhaitait mettre en place une deuxième chambre élective exerçant une fonction modératrice à l'instar de celle exercée dans les bicamérismes fédéraux (advice and consent du bicamérisme américain). Néanmoins, celle-ci n'exercerait nullement de telles fonctions et se placerait dans les bicamérismes non fédéraux, précisément dans les pays ayant une forme de gouvernement parlementaire.

Il est intéressant in fine de remarquer que si le Statut Albertin de I'Italie avait mis en place une rationalisation implicite à son bicamérisme non fédéral, globalement égalitaire relatif, par le fameuses infournades de sénateurs qui s'apparentaient au système du patronage mis en place en Angleterre par le Life PeerAge Act de 1958, les constituants républicains n'ont pas suivi l'exemple de leur prédécesseurs qui, avant l'heure, avaient, par des conventions constitutionnelles, des règles similaires à celles des futurs Parliament Act anglais.

En effet, si le Statut Albertin avait une certaine flexibilité lui permettant de s'adapter à plusieurs situations en raison de son contenu très général, la constitution italienne a voulu préciser les compétences des chambres envers le Parlement. On peut affirmer qu'il s'agit d'une chose tout à fait logique car le Statut Albertin était une constitution flexible, à l'instar de l'actuelle constitution qui est rigide comme la majorité des constitutions contemporaines. Néanmoins, on peut affirmer que la rigidité constitutionnelle n'entrave en rien la possibilité d'être générique sur certains aspects, comme le témoigne la constitution hollandaise qui ne discipline pas formellement le rapport de confiance.

C'est ainsi que si la constitution italienne reste générique quant à la discipline de la procédure législative (art. 70), à l'exception des règles d'examen des textes 
qui découlent des principes classiques de droit parlementaire (art. 72, al. 1), elle discipline de manière précise les rapports de (double) confiance des chambres envers l'exécutif.

Le législateur constitutionnel de 1963 a souhaité aligner la durée des deux chambres, mais n'a pas modifier le régime de confiance. Ce qui aurait pu paraître logique car la constitution italienne contient des indices précieux qui pourraient laisser penser une primauté de la Chambre des députés sur le Sénat.

En premier lieu, les critères différents pour élire les deux chambres, prévus aux articles 48 et 58 de la constitution.

Les dispositions prévues à l'article 58 doivent être mises en lien avec celles prévues à l'article 75 . En effet, concernant le référendum abrogatif, la constitution prévoit que " tous les citoyens appelés à élire la Chambre des députés ont le droit de participer au référendum " (alinéa 3 de l'article 75). C'est ainsi qu'indirectement on peut affirmer que la représentation politique générale a été conféré par les constituants à la Chambre des députés, car participent à l'élection de cette chambre les électeurs qui, aux termes de l'article 48, ont 18 ans.

Cette potentielle supériorité qui pourrait ressortir de l'obiter dictum de l'article 75 alinéa 3 est empêchée par dispositions contenues dans l'article 94.

Ainsi, une révision constitutionnelle éliminant le rapport de double confiance aurait pu être envisagée en raison des dimensions plus vastes de l'électorat qui élit les députés par rapport à celui plus restreint des sénateurs. Critère qui d'ailleurs a été privilégié par les constituants pour déterminer le périmètre des électeurs pouvant participer au référendum abrogatif, mais aussi pour le référendum constitutionnel (art. 138).

Le problème concernant le bicamérisme italien ne réside pas dans sa nature égalitaire absolue (bicamerismo perfetto, selon les termes employés par la doctrine italienne), mais plutôt dans le rôle du Sénat dans le système bicaméral. En effet, on peut affirmer que le bicamérisme est une condition pour réaliser le principe de la démocratie pluraliste car il permet aux différentes composantes pluralistes de participer à l'ensemble des procédures de décisions dans lesquelles l'État compose les différents intérêts et les synthétise en vue de l'intérêt général. Le type de représentation va influer sur les fonctions exercées par les deux chambres. Se pose ainsi un problème sur le rôle du Sénat italien dans la fonction bicamérale, soit sa contribution au pluralisme institutionnel en apportant une intégration à la représentation politique générale exprimée par la première chambre ${ }^{97}$.

Le bicamérisme italien pose un problème car les deux chambres mettent en place le principe de "la division du souverain " qui postule un conflit entre deux assemblées élues au suffrage universel qui représentent, sans distinction dans leur composition, le même corps électoral98.

97 Cf., F. PARLERMO, M. NICOLINI, II bicameralismo, pluralismo e limiti della rappresentanza in prospettiva comparata, Edizioni Scientifiche Italiane, Napoli, 2013, op. cit., p. 222.

98 Ce terme fut employé fut employé en Angleterre lors d'une délibération de la House of Commons 
Ainsi, le ratio du bicamérisme italien est lié au concept de centralité du Parlement qui n'a pas pour réel but constitutionnel celui de valoriser l'institution parlementaire mais "de défendre la centralité du système des partis "99. La formule utilisée pour décrire l'élection du Sénat " élu sur base régionale ", art. 57, alinéa 1 de la constitution), qui pourrait laisser imaginer une fonction d'intégration à la réalité territoriale (celle des Régions), est en réalité une fonction d'intégration accordée aux partis politiques qui, comme pour la Chambre des députés, déterminent la composition d'un Parlement entièrement élu au suffrage universel, où l'élection de la deuxième chambre au suffrage universel met en place un pluralisme politique-partitique, lié aux Régions seulement par une formule constitutionnelle qui a désormais perdu tout son sens : les sénateurs sont élus sur base régionale.

\section{Résumé}

L'article fait l'examen du bicamérisme égalitaire italien à travers d'une analyse comparative des autres bicamérismes présents en Europe et dans le monde. II met en évidence la spécificité de ce bicamérisme unique en son genre. Aucun autre bicamérisme égalitaire ne s'apparente à ce dernier, en raison des compétences identiques exercées par les deux chambres : la fonction législative, la confiance au Gouvernement accordée par les deux chambres. La première de ces deux fonctions est présente dans d'autres pays européens (Suisse, Bosnie-Herzégovine) et extraeuropéen (USA), même si la spécificité italienne consiste en l'absence d'organe permettant d'asseoir a minima une entente sur les textes en discussion entre les deux chambres à travers un organe de conciliation. La deuxième fonction est une spécificité de ce bicamérisme qui permet de constater un de ces atouts principaux: un bicamérisme non rationalisé. En outre, ces deux fonctions permettent d'observer un bicamérisme indifférencié, les deux chambres ont non seulement les mêmes compétences et quasiment la même composition. La seule différence réside dans une formule prévue dans la Constitution (article 57, al. 1) qui a désormais perdu tout son sens "Le Sénat est élu sur base régionale ", permettant d'apparenter les deux assemblées à deux jumeaux monozygotes.

de 2007 (Cf., House of Commons Hansard, 27 june 2011, c.677 e 679 ; Cf. M. RUSSELL, Elected Second Chambers and their Powers : An International Survey, dans Pol.Quaterly, 2012,83 (1), cit., p. 117) pour mettre en place une "largely or wholly elected chamber". En réponse à cette délibération se prononça le Joint Commitee déclarant que "If the Lords Acquired an electoral mandate... their relationship with the Commons, would inevitably be called into question". Cf., Joint Committee on Conventions, Convention of the UK Parliament, London, Houses of Parliament, 2006, rappellé par M. RUSSEL, Elected Second Chamber, op. cit., p. 117.

99 Cf., G. RIVOSECCHI, Art. 55, in R. BIFULCO, A. CELOTTO, M. OLIVETTI (a cura di) Commentario alla Costituzione. Art. 55-100, t.1, UTET, Torino, 2006, p. 1099. 


\begin{abstract}
The article examines Italian egalitarian bicameralism through a comparative analysis of other examples of egalitarian bicameralism in Europe and worldwide. It highlights the specific nature of this bicameralism, which is a unicum. No other egalitarian bicameralism is similar to this perfect egalitarian bicameralism, owing to the competences exercised in an identical form by the two chambers: the legislative function, and the confidence in the Government granted by the two chambers. The first of these two functions is present in other European countries (Switzerland, Bosnia-Herzegovina) and outside Europe (USA), although the specific nature of the Italian model lies in the absence of a body allowing a minimum agreement on the texts under discussion to be established between the two chambers through a conciliation body. The second function is specific to this bicameralism which reveals one of its main assets: an unstreamlined bicameralism. Moreover, these two functions reveal an undifferentiated bicameralism, with both chambers having not only the same competences but also almost the same composition. The only differentiating element lies in a formula in the Constitution (Article 57, para. 1) which has now lost all meaning: "The Senate is elected on a regional basis", meaning that the two assemblies can be compared to two homozygous twins.
\end{abstract}

